\title{
A Systems Pharmacology Approach for Identifying the Multiple Mechanisms of Action for the Rougui-Fuzi Herb Pair in the Treatment of Cardiocerebral Vascular Diseases
}

\author{
Chun Li, ${ }^{1}$ Xia Du, ${ }^{2}$ Yang Liu, ${ }^{2}$ Qi-Qi Liu, ${ }^{1}$ Wen Bing Zhi, ${ }^{2}$ Chun Liu Wang, ${ }^{2}$ Jie Zhou, \\ Ye Li $(\mathbb{D})^{2}$ and Hong Zhang $\mathbb{( i D}^{2}$ \\ ${ }^{1}$ Pharmacy College, Shaanxi University of Chinese Medicine, Xianyang, Shaanxi, China \\ ${ }^{2}$ Shaanxi Academy of Traditional Chinese Medicine, Xi'an, Shaanxi, China \\ Correspondence should be addressed to Ye Li; liye_11@163.com and Hong Zhang; zhanghong919919@163.com \\ Chun Li and Xia Du contributed equally to this work.
}

Received 25 October 2019; Revised 5 December 2019; Accepted 12 December 2019; Published 13 January 2020

Academic Editor: Carmen Mannucci

Copyright ( $\odot 2020$ Chun Li et al. This is an open access article distributed under the Creative Commons Attribution License, which permits unrestricted use, distribution, and reproduction in any medium, provided the original work is properly cited.

\begin{abstract}
Cardiocerebral vascular diseases (CCVDs) are the main reasons for high morbidity and mortality all over the world, including atherosclerosis, hypertension, myocardial infarction, stroke, and so on. Chinese herbs pair of the Cinnamomum cassia Presl (Chinese name, rougui) and the Aconitum carmichaelii Debx (Chinese name, fuzi) can be effective in CCVDs, which is recorded in the ancient classic book Shennong Bencao Jing, Mingyibielu and Thousand Golden Prescriptions. However, the active ingredients and the molecular mechanisms of rougui-fuzi in treatment of CCVDs are still unclear. This study was designed to apply a system pharmacology approach to reveal the molecular mechanisms of the rougui-fuzi anti-CCVDs. The 163 candidate compounds were retrieved from Traditional Chinese Medicine System Pharmacology Database and Analysis Platform (TCMSP). And 84 potential active compounds and the corresponding 42 targets were obtained from systematic model. The underlying mechanisms of the therapeutic effect for rougui-fuzi were investigated with gene ontology (GO) enrichment analysis and Kyoto Encyclopedia of Genes and Genomes (KEGG) pathway enrichment analysis. Then, component-target-disease (C-T-D) and target-pathway (T-P) networks were constructed to further dissect the core pathways, potential targets, and active compounds in treatment of CCVDs for rouguifuzi. We also constituted protein-protein in interaction (PPI) network by the reflect target protein of the crucial pathways against CCVDs. As a result, 21 key compounds, 8 key targets, and 3 key pathways were obtained for rougui-fuzi. Afterwards, molecular docking was performed to validate the reliability of the interactions between some compounds and their corresponding targets. Finally, UPLC-Q-Exactive-MS ${ }^{\mathrm{E}}$ and GC-MS/MS were analyzed to detect the active ingredients of rougui-fuzi. Our results may provide a new approach to clarify the molecular mechanisms of Chinese herb pair in treatment with CCVDs at a systematic level.
\end{abstract}

\section{Introduction}

Cardiocerebral vascular diseases (CCVDs), including thrombosis, atherosclerosis, hypertension, cardiac hypertrophy, myocardial infarction, heart failure, stroke, and cerebral ischemia, are the major health problem all over the world, claiming more than 17.3 million lives annually [1-3]. Given the high incidence and high mortality of CCVDs, seeking feasible prevention and treatment strategy is highly imperative and critical to human health $[4,5]$. Western medicine is currently the mainstream medical treatment for
CCVDs. However, some certainly existing ill-effects are still unavoidable. For example, some antihypertensive drugs have triggered serious side effects, with hot flush, fatigue, shortness of breath, headache, dizziness rhabdomyolysis, and hepatic diseases [6-8]. Therefore, it is very indispensable to seek better, safe, and effective treatment strategies in treatment of CCVDs.

Herb pairs, the simplest form and the centralized representative of Chinese herbal compatibility, intrinsically convey the basic idea of traditional Chinese medicines' prescriptions that has attracted comprehensive attention 
for their ability to treat complex and chronic diseases due to their moderate treatment effect and lower side effect [9-11]. The herb pair (rougui-fuzi) consists of the twigs of Cinnamomum cassia Presl (Chinese name, rougui) and the root of Aconitum carmichaelii Debx (Chinese name, fuzi) that can be effective in the treatment of CCVDs, and usually in a proportion of $1: 1$, according to the classic ancient book Shennong Bencao Jing, Mingyibielu and Thousand Golden prescriptions [12]. Among them, the herb Cinnamomum cassia Presl can increase coronary blood flow, improve coronary circulation and myocardial nutritional status, and was often used in the prevention and treatment of coronary heart, arrhythmia, rheumatic heart disease, cardiogenic hypertension, and cardiovascular diseases [13-16]. Aconitum carmichaelii Debx has the functions of protecting cardiac muscle cell, fight arrhythmia, decrease inflammatory reaction, and inhibit tumor. Moreover, several active constituents of these two herbs are documented to exhibit various biological activities, which contribute to the CCVDs. For example, cinnamaldehyde, an index component of the Cinnamomum cassia Presl, can dilate peripheral blood vessels, improve blood circulation at the end of blood vessels, and improve myocardial blood supply. The aconite polysaccharide could increase the synthesis of metallothionein, reduce the production of malondialdehyde (MDA) and the release of lactate dehydrogenase (LDH), and inhibit cardiomyopathy apoptosis [17]. As is demonstrated above, the herb pair rougui-fuzi plays an important role in the treatment of CCVDs, but molecular mechanisms of rougui-fuzi in treatment of CCVDs are still indecisive.

Systems pharmacology has become a powerful tool to systematically reveal the function and behavior of a complex biological system, and it has become a novel and efficient way to explore the pharmacological mechanism of traditional Chinese medicine [18, 19]. It likewise has been commonly presented lately for exploring the molecular mechanisms of traditional Chinese medicine and different complex chronic health conditions, including CCVDs, cancer, and metabolic illness $[20,21]$. It is more remarkable that systems pharmacology was also used to reveal the therapeutic mechanism of herb pairs in treatment with disease. For example, the action of herb pair (dangguihonghua) in treatment of blood stasis syndrome (BBS) through interactions between multigenes and multicompounds, instead of directly acting on either single target or single constituent, was to successfully elucidate the polypharmacological mechanisms underlying the efficiency of danggui-honghua for BSS treatment based on systems pharmacology [22]. Considering the herbal constituents and their related targets based on systems pharmacology analysis can enable us to better comprehend the molecular mechanisms of the herb pair rougui-fuzi [23].

In this study, information on constituents and corresponding candidate targets of rougui-fuzi was acquired from TCMSP databases. Then, the related biological processes and signal pathways were obtained according to GO and KEGG enrichment analysis. Moreover, compound-target-disease (C-T-D) and target-pathway (T-P) networks were established. Meanwhile, protein-protein interaction (PPI) was mapped using important pathway-related targets for screening core target proteins based on (STRING) database. After that, molecular docking was carried out to verify the reliability of compound-target binding interactions. Finally, to further determine rougui-fuzi components, the UPLC-QExactive-MS ${ }^{\mathrm{E}}$ and GC-MS/MS analyses were performed (the whole flowchart has been shown in Figure 1). Overall, this work may provide a useful system pharmacology framework to interpret multicomponent, multitarget, multichannel mechanism for the herb pair rougui-fuzi, and may give some basis and enlightenment in the further research for the herb pair.

\section{Materials and Methods}

\subsection{Compound Database Construction for Rougui-Fuzi.} The constituents of medicinal herbs Aconitum carmichaelii Debx and Cinnamomum cassia Presl were extracted from the Traditional Chinese Medicine System Pharmacology Database and Analysis Platform (TCMSP, http://lsp.nwu.edu.cn/ tcmsp.php) [24, 25]. TCMSP is a unique system pharmacology platform designed for herbal medicines, which provides up-to-date, quantitative, and accurate structural and physicochemical properties such as drug targets and their relationships with diseases. Meanwhile, a large-scale structural information $(29,384$ chemicals in total with 13,144 unique molecules) with manually curated information for all recorded herbs in Chinese pharmacopoeia was integrated [26].

2.2. Target Fishing. Drug target indentifying is momentous to elucidating the biological basis of traditional Chinese medicine. Thus, in this work, we applied a systematic model that efficiently enriched chemical, genomic, and pharmacological information for drug target, based on random forest (RF) and support vector machine (SVM) methods. The robust model showed optimal performance of predicting the drug-target interactions, with $82.83 \%$ concordance, $81.33 \%$ sensitivity, and $93.62 \%$ specificity, which calculated the possibility of interactions between each ingredient and its target from TCMSP [27, 28]. There is a nonstandard problem in the naming of compound targets searched in the database; so, all TCMSP drug targets are imported into the UniProt (https://www.uniprot.org/) database, the target gene name is entered to define the species as "Homo sapiens," and all protein names are corrected to their official name (official symbol). The human CCVD-related diseases associated with genes were secured from the curative targets database such as the Online Mendelian Inheritance in Man (OMIM, http:// www.omim.org/) and Pharmacogenomics Knowledgebase (PharmGKB; http://www.pharmgkb.org) [29-32]. The keywords were "stroke," "anti-platelet aggregation," "atherosclerosis," "improving microcirculation," and "antithrombin" [33-35].

Based on the above, the previous compound targets are mapped with the genes of human CCVD-correlative disease 


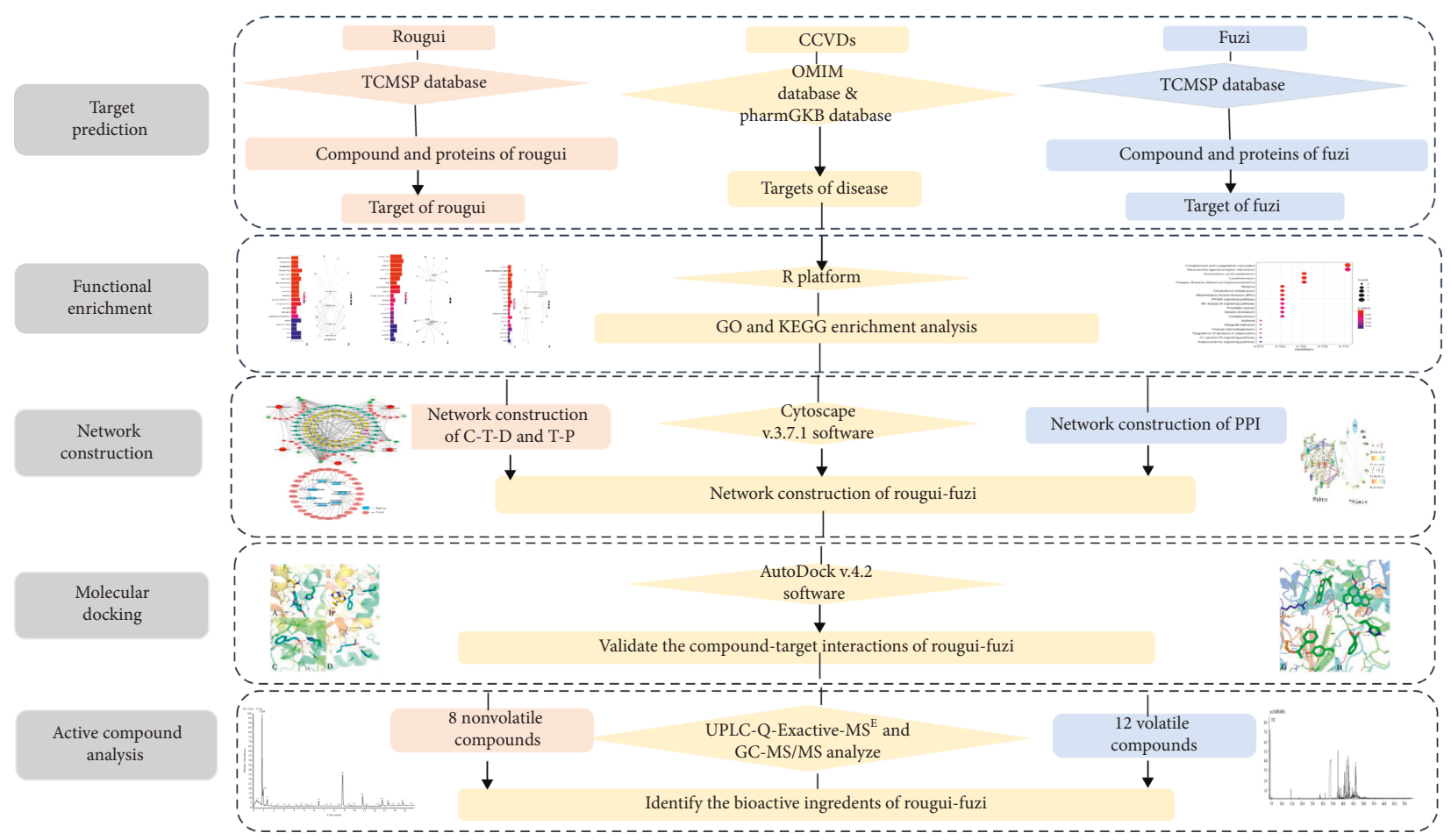

FIGURE 1: Flowchart of the experimental procedures.

to obtain their common targets, for better deciphering the role of rougui-fuzi against CCVDs.

2.3. Functional Enrichment Analysis. To better comprehend the potential biological processes and pathways of rouguifuzi in treatment of CCVDs, the Kyoto Encyclopedia of Genes and Genomes (KEGG) pathway (http://www.genome. jp/kegg/pathway.html) and gene ontology (GO) enrichment analyses were performed based on the ClusterProfiler software package on $R$ platform, and compared with $\mathrm{Vi}$ sualization [36]. Afterwards, the GO interactive network and the bubble diagram of KEGG pathways were structured based on the topGO packet of $R$ platform [37]. $P$ value was calculated in these two enrichment analyses, and $P<0.05$ suggested the enrichment degree of statistically significant [38]. As a result, the pathway association and GO functions based on their enrichment were found and appropriately described.

2.4. Network and Protein-Protein Interaction Data Construction. For better dissecting the potential molecular mechanism of rougui-fuzi, we established three corresponding networks: (1) compound-target-disease (C-T-D) network. Active constituents of rougui-fuzi, corresponding targets, and CCVD-related diseases were employed to generate the C-T-D network in which an ingredient and a target are connected with each other if this protein is a known or validated either target or disease of this molecule. (2) Target-pathway (T-P) network. We extracted the precise pathway information of targets from the database of KEGG, and then constructed a target-pathway bipartite graph that comprises targets and their corresponding normative pathways. The previous two were generated in Cytoscape 3.7.2 software that is a standard tool for biological network visualization and data integration to further analyze the mutual relations in the network $[37,39]$. (3) Protein-protein interaction (PPI network) was mapped using important pathway-related targets for screening core target proteins based on (STRING) database (https://string-db.org/). The version 11.0 of STRING was employed to seek for the PPI data, with the species limited to "Homo sapiens" and a confidence score $>0.4$ [40]. Meanwhile, the node 1, node 2, and combined score of the STRING database were imported into Cytoscape3.7.1 software, with setting the node size and color to map the degree value and score value to construct PPI network [41].

2.5. Molecular Docking. To verify the reliability of compound-target binding interactions, molecular docking program was in progress using AutoDock software (version 4.2), which employs the Lamarckian genetic algorithm (LGA) for each progress [42]. All the 3D structures of target proteins were downloaded from the RCSB PDB database (http://www.pdb.org), so that proteins and ligands could be prepared in the AutoDock tools before performing the docking progress [43]. The auxiliary program Autogrid was used to generate the docking area, which was defined as a $60 \times 60 \times 603 \mathrm{D}$ grid centered around the ligand-binding site with a $0.375 \AA$ grid space. All bond rotations for the ligands were ignored in this study. Finally, the docking binding energy was calculated to explore binding interactions 
between the compounds and their targets in our studies, the potential protein targets of TCM were successfully predicted with a good compound-protein binding affinity based on the threshold level of $-5.0 \mathrm{kcal} / \mathrm{mol}$ [43].

2.6. UPLC-Q-Exactive-MSE Analysis of Rougui-Fuzi. To verify the potential ingredients of rougui-fuzi, the UPLC-QExactive-MS ${ }^{\mathrm{E}}$ analysis was applied. Some reagents were also used: rougui (batch no. 19061412) was provided by Shaanxi Kangyu Pharmaceutical Co., Ltd (Xi'an, China), and fuzi (batch no. 150816) was provided by Shaanxi Kangyu Pharmaceutical Co., Ltd (Xi'an, China). LC-MS-grade acetonitrile was obtained from Thermo Fisher Scientific (China) co., ltd. (Shanghai, China), MS-grade formic acid was obtained from Thermo Fisher Scientific (China) co., ltd. (Shanghai, China). The rougui-fuzi extracts were analyzed on a Thermo Fisher Scientific quadrupole-electrostatic field orbital well high-resolution system coupled with $\mathrm{H}$-Class UPLC system. Separations were accomplished on an Accucore AQ C18 column $(150 \mathrm{~mm} \times 2.1 \mathrm{~mm}, 2.6 \mu \mathrm{m})$. The mobile phase included $0.1 \%$ formic acid aqueous solution (Solvent A) and acetonitrile (Solvent B) and a gradient elution program was performed: $0 \mathrm{~min}, 98 \%$ solvent A; $17 \mathrm{~min}, 50 \%$ solvent A; $20 \mathrm{~min}, 98 \%$ solvent $\mathrm{A}$. The flow rate was set at $0.3 \mathrm{~mL} / \mathrm{min}$. Column temperature was kept at $30^{\circ} \mathrm{C}$ and the total run time was $20 \mathrm{~min}$. The autosampler was conditioned at $4^{\circ} \mathrm{C}$ and the injection volume was $1 \mu \mathrm{L}$. The ion source of thermoelectric spray (HESI) mass spectrometry was used to collect data in full switch ion mode. The sheath gas flow rate was set $30 \mathrm{~L} / \mathrm{min}$, spray voltage was set $3.50 \mathrm{kV}$, and capillary temperature and aux gas heater temperature were $300^{\circ} \mathrm{C}$. S-lens $\mathrm{RF}$ level was set $55 \mathrm{~V}$. The scan mode was Full MS and dd-MS ${ }^{2}$, Full MS resolution of the detection mode was 7000, and the dd-MS ${ }^{2}$ resolution was 175000. The scan range was from 100 to $1200 \mathrm{Da}$. Leucineenkephalin was used as the lock mass for generating reference ions in the positive and negative modes.

2.7. GC-MS/MS Analysis of Rougui-Fuzi. The rougui-fuzi extracts were analyzed on Shimadzu TQ8040 coupled with Shimadzu 2010Plus GC system. Separation was performed on a $19091 \mathrm{~s}-433 \mathrm{HP}-5 \mathrm{MS}(30 \mathrm{~m} \times 0.25 \mathrm{~mm}$ ID with $0.25 \mu \mathrm{m})$ capillary column. The inlet temperature was set at $260^{\circ} \mathrm{C}$; the carrier gas (helium; purity $>99.996 \%$ ) was maintained at a constant flow of $1.0 \mathrm{~mL} \cdot \mathrm{min}^{-1}$; the mode of inlet was split less; the injection volume was $1 \mu \mathrm{L}$; the temperature of column oven was programmed from an initial value of $60^{\circ} \mathrm{C}$ (hold for $10 \mathrm{~min}$ ), and then raised at $4^{\circ} \mathrm{C} \cdot \mathrm{min}^{-1}$ up to $220^{\circ} \mathrm{C}$ (hold for $20 \mathrm{~min}$ ); the split ratio was set $20: 1$.

\section{Result and Discussion}

3.1. Ingredient Collection of Rougui-Fuzi. In this research, a total of 165 compounds were candidate compounds extracted in rougui-fuzi, including 65 kinds in fuzi and 100 kinds in rougui. Among the 165 compounds, 2 were duplicated and therefore removed, resulting in a list of 163 components. Through observation and analysis, it was found that fuzi mainly includes alkaloids, flavonoids, polysaccharides, sterol, and organic acid [44]. Rougui mainly includes volatile oils, polyphenols, flavonoids, polysaccharides, lignin class, and diterpenes [14].

3.2. Target Prediction and Disease Mapping. In this study, a total of 194 genes were linked to the 163 identified compounds of the herb pair by TCMSP database. Among them, 65 ingredients in fuzi act together on 58 targets, 100 compounds in rougui act together on 181 targets, and there are 45 common targets in rougui-fuzi. It is potently demonstrated that the same active ingredients can act on different targets, and different active constituents can also act on the same target, reflecting its multicomponent, multitarget anti-CCVD mode of action. Then, through the OMIM and pharmGKB databases, 158 targets obtained were mapped to stroke-related drug targets, 547 targets obtained were reflected to antiplatelet aggregation-related drug targets, 232 targets obtained were mirrored to antiatherosclerosis-related drug targets, 479 targets obtained were reflected to improve microcirculation-related drug targets, and 79 targets obtained were mirrored to antithrombinrelated drug targets. Finally, 84 potential active compounds (see Table S1) and corresponding 42 targets (see Table S2) were identified after deduplication for rougui-fuzi in treatment with CCVDs.

\subsection{GO Term and KEGG Pathway of Potential Target Gene Enrichment Analysis}

3.3.1. GO Enrichment Analysis. The results of GO evaluation were illustrated by the biological process (BP), cell component (CC), and molecular function (MF) terms. In the $R$ package of clusterProfiler, 30 terms of BPs, 30 terms of CCs, and 25 terms of MFs enriched for these potential targets were recognized as $P<0.05$. Depending on the outcomes of GO enrichment, the enriched BP ontologies were dominated by positive regulation of blood coagulation, positive regulation of hemostasis, positive regulation of wound healing, and regulation of response to wounding, indicating that the active components of rougui-fuzi interact primarily with related targets in the positive regulation (see Figure 2). The enriched CC ontologies were dominated by secretory granule, cytoplasmic vesicle lumen, vesicle lumen, external side of plasma membrane, and side of membrane (see Figure 3). The enriched MF ontologies were dominated by nuclear receptor activity, serine hydrolase activity, receptor ligand activity, transcription factor activity, direct ligandregulated sequence-specific DNA binding, and steroid hormone receptor (see Figure 4).

3.4. KEGG Pathway Annotation Analysis. The results showed that 42 targets were mapped to a total of 19 pathways $(P<0.05)$ through the bubble diagram (see Figure 5). Next, we excluded the CCVD-independent pathways such as "leishmaniasis," "malaria," and "inflammatory bowel disease," and selected the most potential target enrichment of 


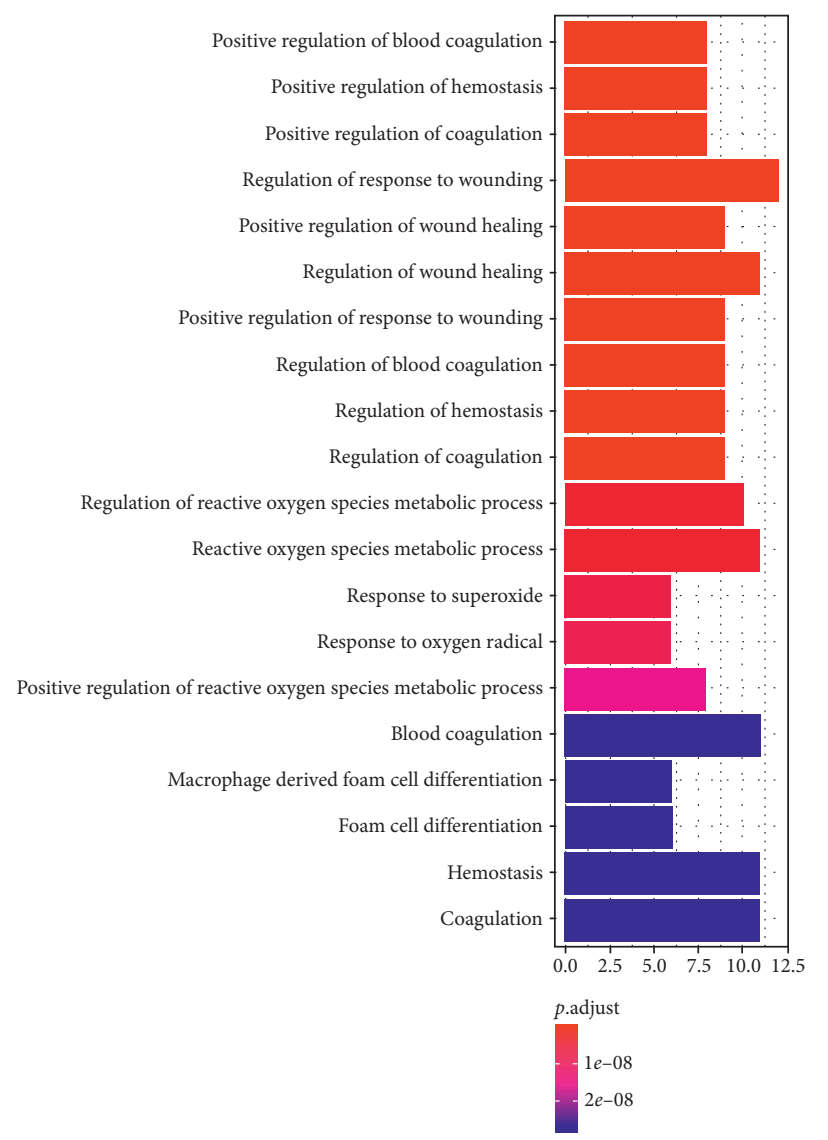

(a)

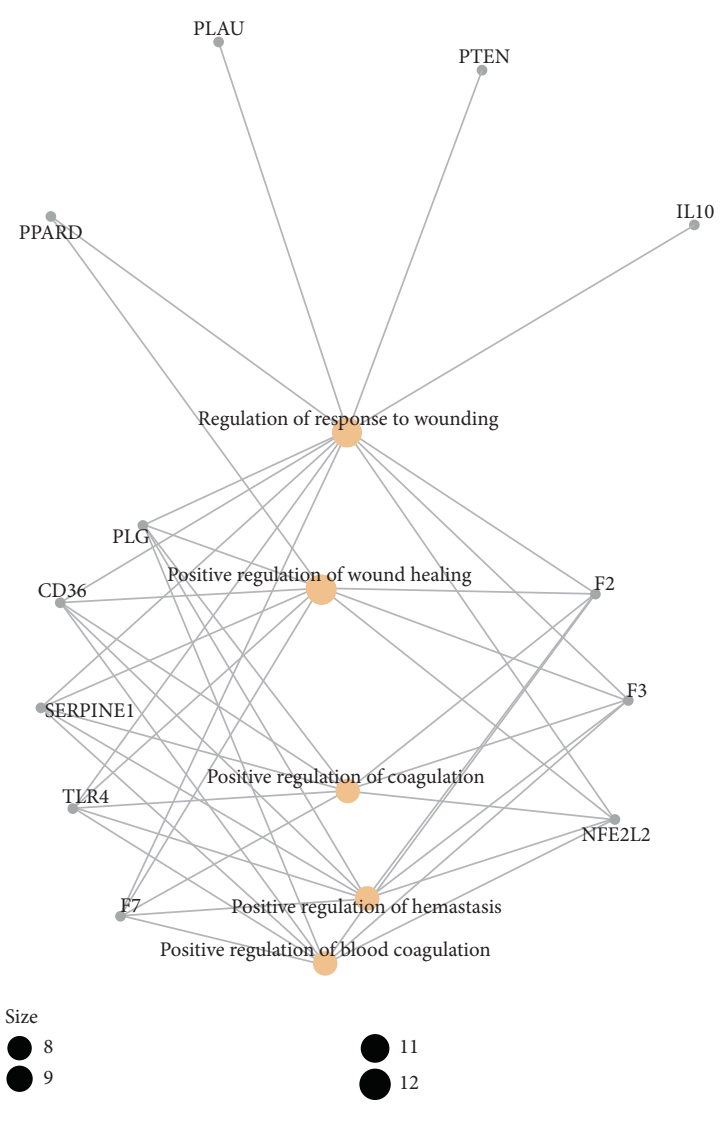

(b)

FIgURE 2: Biological process (BP) analysis of the predicted targets in rougui-fuzi. (a) The 30 GO terms for BP ontology with the most significant $P$ values of targets in CCVDs. (b) The network diagram of core target regulation pathway.

the pathways for analysis. It was not difficult to find that complement and coagulation cascades, neuroactive ligand-receptor interaction, and arachidonic acid metabolism are relatively significant pathways. Even though PI3K-Akt signaling pathway is not present in Figure 5, it plays an important role in the treatment of cardiovascular disease, and, the 6 differentially expressed genes involved in PI3K-Akt signaling pathway are as follows: IFNB1, IL4, INS, PTEN, RXRA, and TLR4, which have a crucial role in CCVD-related diseases [45, 46]. As a result, 7 pathways were determined in treatment of CCVDs.

3.5. Network Construction and Analysis. Centrality is a universal used notion in network analysis to express the significance of a node throughout the network. This work mainly measures the degree centrality (DC), betweenness centrality (BC), and closeness centrality (CC). The DC is the sum of the number of direct connections between a node and other nodes. It is the most direct metric to characterize the importance of nodes in network analysis. CC is the sum of the distances of a node to all other nodes, reflecting the extent of proximity of a node to other nodes. Betweenness centrality is the number of shortest paths passing through a node, reflecting the extent of cohesion of the nodes in the network. The greater the number of the shortest paths passing through a node, the higher its value [47].

3.6. Compound-Target-Disease Network and Analysis. In this work, C-T-D network was constructed including 131 nodes (84 active compounds, 42 potential targets, and 5 CCVDrelated diseases) and 293 edges (the interactions between different nodes) (see Figure 6). The yellow circle represented the ingredients of rougui, the blue circle represented the constituents of fuzi, and the purple circle represented the common compounds of the rougui-fuzi. The pink circles represented potential targets of all compound in rougui and fuzi, the green circle the common targets the compound of the rougui-fuzi, and the red circle denoted the CCVD-related diseases. The results indicated that the active compounds had an average DC value of 2.7619, an average CC of 0.3464 , and an average BC of 0.0068. Among them, the three centralities of 21 compounds were all higher than the average, indicating that these compounds have a relatively important status in the network (see Table 1). Here, oleic acid is one of the core components of rougui-fuzi and displays higher centralities of target interactions $(D C=17$, $C C=0.4577, B C=0.1394)$. It has been reported as a potential 


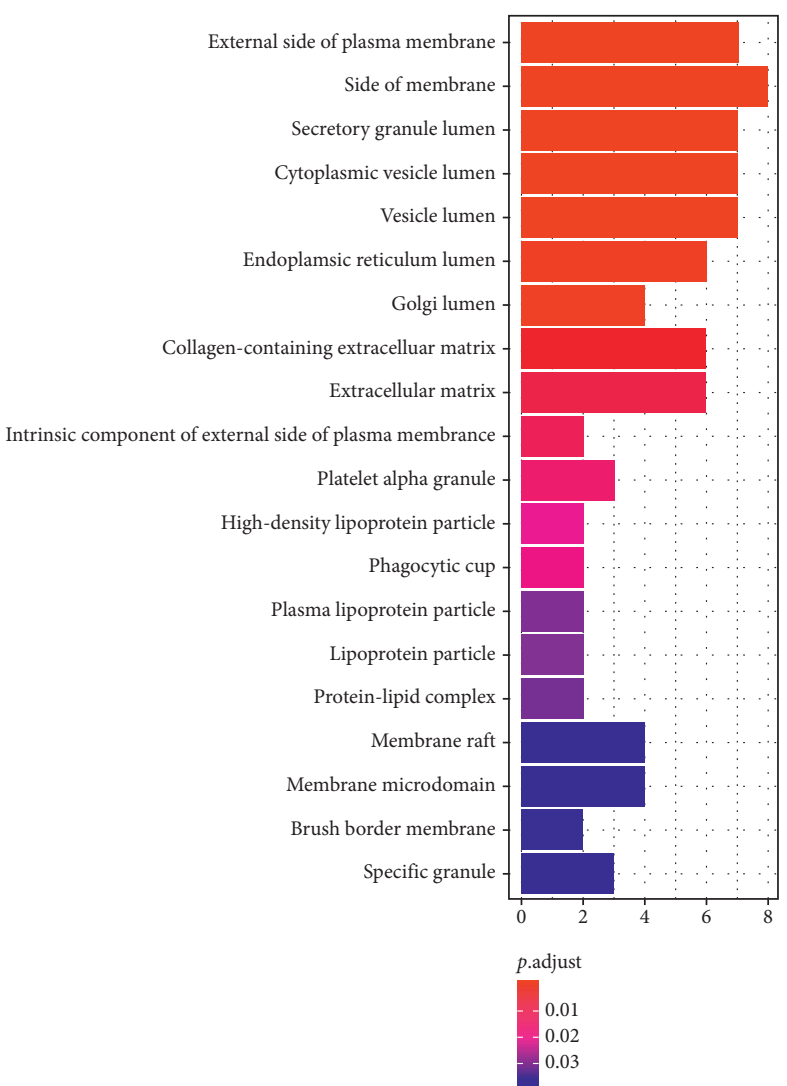

(a)

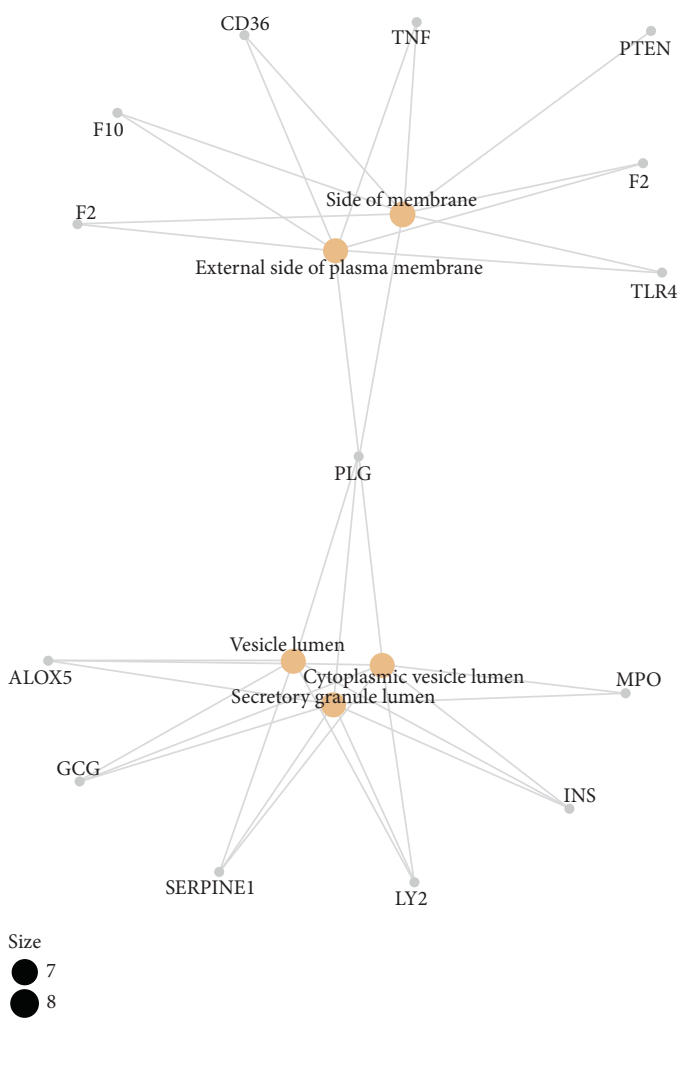

(b)

Figure 3: Cellular component (CC) analysis of the predicted targets in rougui-fuzi. (a) The 30 GO terms for CC ontology with the most significant $P$ values of targets in CCVDs. (b) The network diagram of core target regulation pathway.

preventive and therapeutic CCVD safety reagent based on some experiments [48, 49]. In addition, palmitic acid [50] $(\mathrm{DC}=6, \mathrm{CC}=0.0332, \mathrm{BC}=0.3779)$, eugenol $[51] \quad(\mathrm{DC}=5$, $\mathrm{CC}=0.0297, \quad \mathrm{BC}=0.4305)$, coumarin $[52,53] \quad(\mathrm{DC}=5$, $\mathrm{CC}=0.0127, \mathrm{BC}=0.3757)$, and linoleic acid $[54] \quad(\mathrm{DC}=4$, $\mathrm{CC}=0.0181, \mathrm{BC}=0.4140$ ) also play an important role in anti-CCVDs.

3.7. Target-Pathway Network and Analysis. In this study, the target-pathway (T-P) network was constructed including 36 nodes (29 potential targets and 7 CCVD-related pathways) and 41 edges (see Figure 7). The pink ellipse represented the targets, and the blue rectangle represented the significant pathways of rougui-fuzi in treatment with CCVDs. The results indicated that the inherent pathways had an average DC value of 5.1429, an average CC of 0.2141 , and an average $\mathrm{BC}$ of 0.3818 (see Table 2). Among them, we found the complement and coagulation cascades pathway $(\mathrm{DC}=7$, $\mathrm{CC}=0.2381, \mathrm{BC}=0.5017)$ and PI3K-Akt signaling pathway $(\mathrm{DC}=6, \mathrm{CC}=0.2518, \mathrm{BC}=0.5513)$ showed higher centrality in the target-pathway network. Meanwhile, according to copious literature, arachidonic acid metabolism, complement and coagulation cascades, PI3K-Akt signaling pathway, NF-kappa B signaling pathway, PPAR signaling pathway, cholesterol metabolism, and neuroactive ligandreceptor interaction play a significant role in CCVDs.
As a critical signaling pathway, complement and coagulation cascades involved in 7 differentially expressed genes, including F10, F3, F2, SERPINE1, F7, PLG, and PLAU. The complement and coagulation systems are two related protein cascades in plasma that serve important roles in host defense and hemostasis, respectively [55]. Recently, it has been shown that hyperactive complement is referred to the pathogenesis (including thrombosis) of diseases such as paroxysmal nocturnal hemoglobinuria, atypical haemolytic uremic syndrome, antiphospholipid syndrome, and bacteremia [56]. Upon vascular injury, tissue factor (TF) is exposed to the blood. TF exposure can initiate a series of positive feedback resulting in thrombin generation and fibrin formation, such as F3 (tissue factor) can activate F2 (thrombin) by Fxa (coagulation propagation). Ultimately, this leads to generation of fibrin, and results in the occurrence and aggravation of thrombosis. Rougui-fuzi may be used to prevent and treat CCVDs by inhibiting F2 and F3. Additionally, serine protease inhibitors (SERPINs) rapidly and irreversibly inhibit the coagulation enzymes and in concert with protein $\mathrm{C}$ system, ultimately terminate the coagulant response [57], which was also consistent with the rougui-fizi anti-CCVDs by inhibiting complement and coagulation cascades pathways.

Using pathway enrichment analysis, we found 5 differentially expressed genes involved in arachidonic acid 


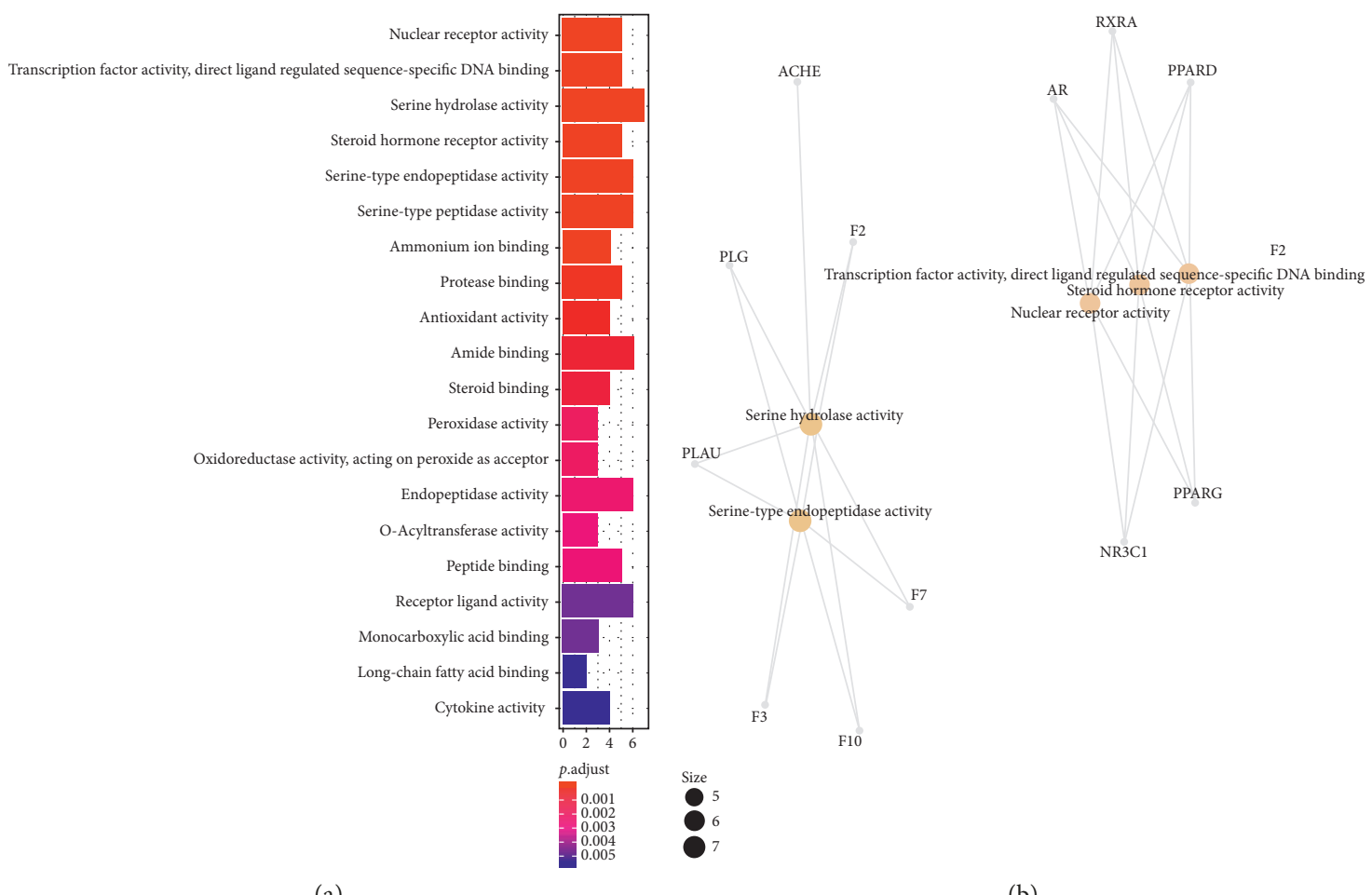

(a)

(b)

FIgUre 4: Molecular function (MF) analysis of the predicted targets in rougui-fuzi. (a) The 25 GO terms for MF ontology with the most significant $P$ values of targets in CCVDs. (b) The network diagram of core target regulation pathway.

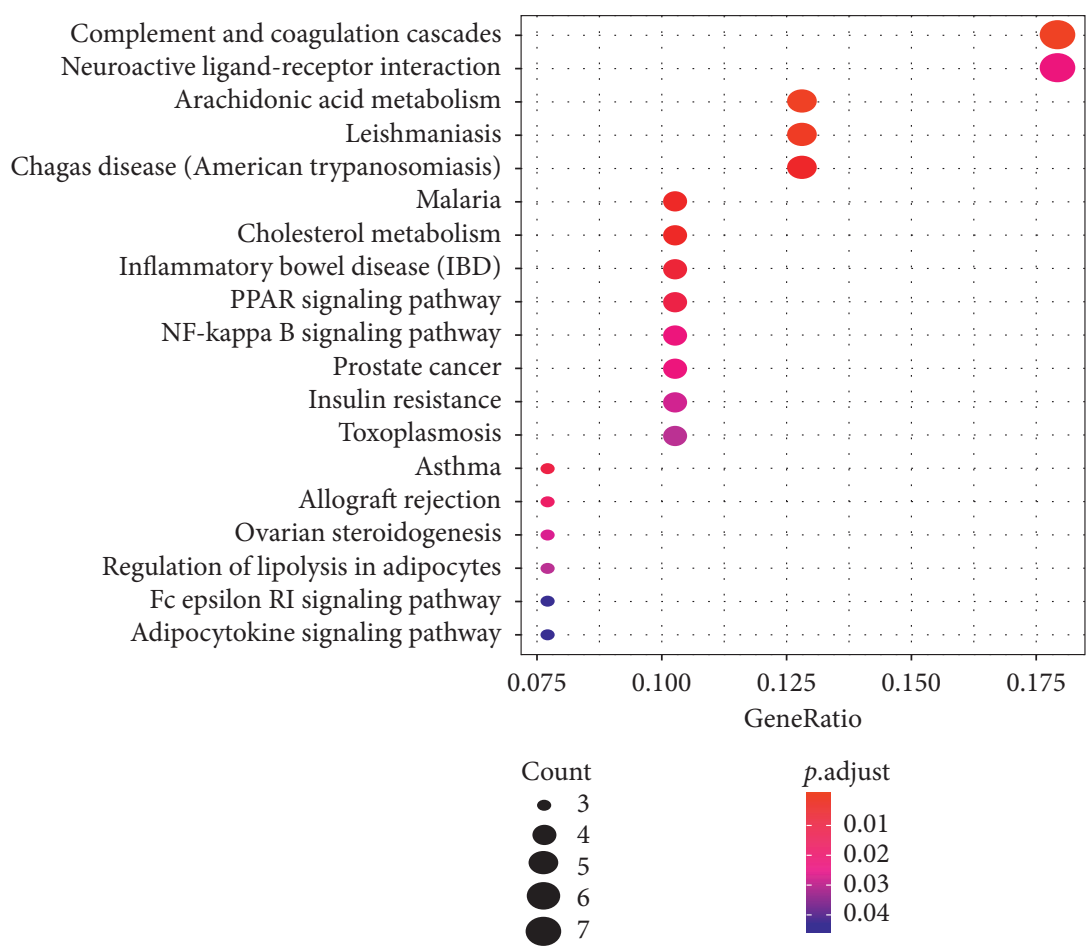

FIGURE 5: KEGG pathway annotation analysis of the rougui-fuzi predicted targets. Bubble diagram of the top 19 KEGG pathways.

metabolism, such as PTGS2, PTGS1, PLA2G2A, LTA4H, and ALOX5. The arachidonic acid (ARA) metabolic network produces a large family of inflammatory mediators, including leukotrienes (LTs) and prostaglandins (PGs), which contribute to numerous inflammatory-related diseases such as asthma and atherosclerosis. Cyclooxygenase 


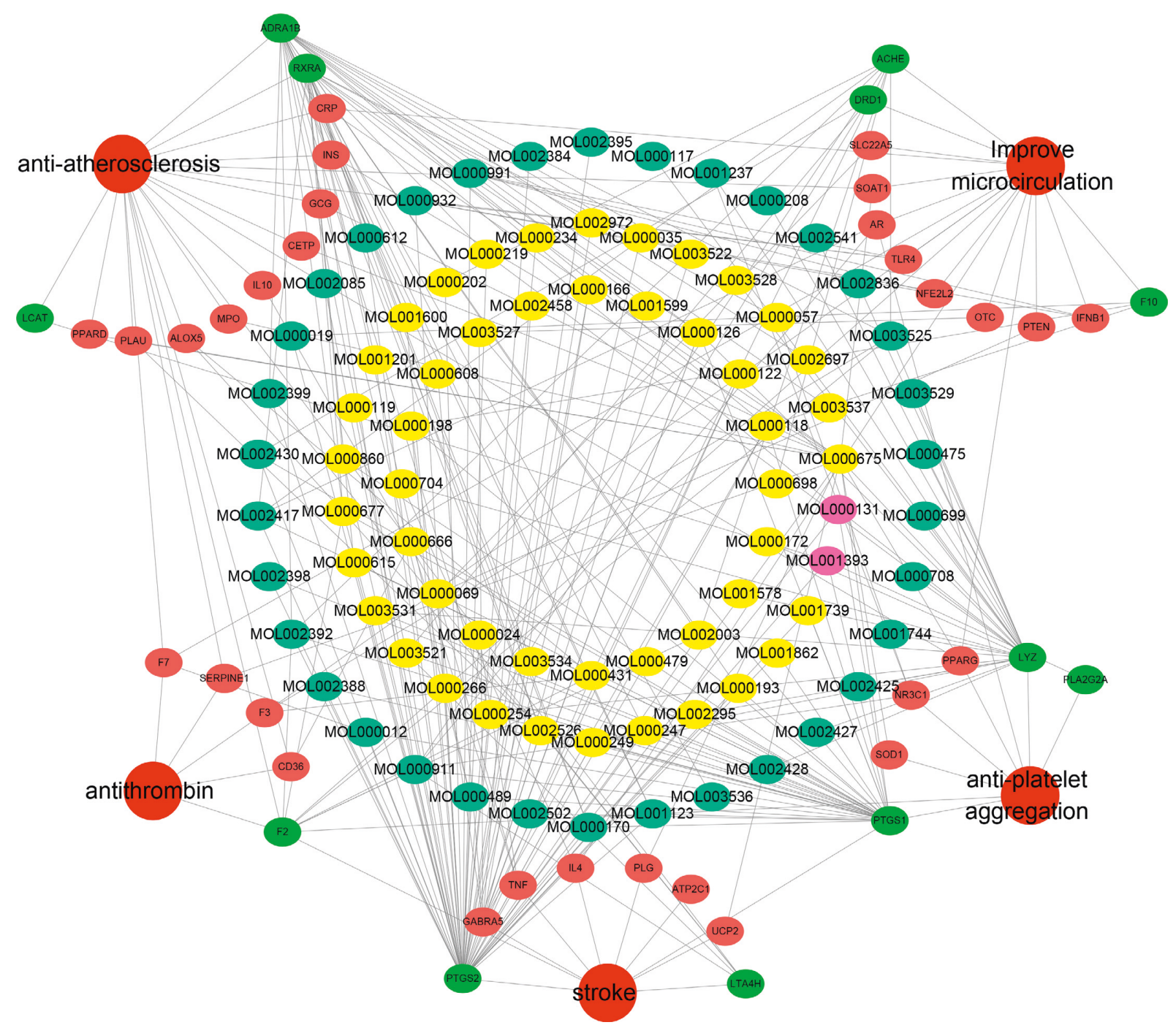

FIGURE 6: Compound-target-disease (C-T-D) network in treatment of CCVDs for rougui-fuzi. The yellow circle represented the ingredients of rougui, the blue circle represented the constituents of fuzi, and the purple circle represented the common compounds of the rougui-fuzi. The pink circle represents potential targets of all compounds in rougui and fuzi, the green circle the common targets the compound of the rougui-fuzi, and the red circle denoted the CCVD-related diseases.

enzymes work in one arm of the pathway to produce prostaglandins (PGs), while the actions of 5-lipoxygenase (ALOX5) and its associated protein work in the other arm of the metabolic pathway to produce leukotrienes (LTs) [58]. After conducting a literature search, the only 5 -LOX inhibitor is zileuton (trade name Zyflo) in clinical trials for treating CCVDs. Inhibitors of LTA4H have also been developed [59]. We presumed that during CCVDs, the production of LTA4H, PTGS2, and PTGS1 was decreased by inhibiting ALOX5, and rougui-fuzi protected against CCVD damage by inhibiting these pathways [60].

In addition, PI3K-Akt signaling pathway referred to 6 differentially expressed genes, including IFNB1, IL4, INS, PTEN, RXRA, and TLR4, and the PI3K-Akt signaling pathway is an important signaling pathway involved in the neuroprotection against ischemic brain damage [61]. Some studies have shown that activation of the PI3K/Akt signaling pathway is related to the protection of ischemia/reperfusion damage in cardiomyocytes [61, 62]. Among them, suppressing PTEN provides protection against ischemic neuron death through both the enhancement of Akt activation and the inhibition of NR2B subunit-containing N-methyl-daspartate receptors [63]. PTEN inhibitors are administered prior or after experimental stroke used to acute neuroprotection following cerebral ischemia [64]. Therefore, we speculated that rougui-fuzi might be anti-CCVD by inhibiting PI3K-Akt signaling pathway.

Consequently, it was concluded that rougui-fuzi may mainly fight CCVDs through complement and coagulation cascades, arachidonic acid metabolism, and PI3K-Akt signaling pathway.

3.8. Protein-Protein in Interaction (PPI) Data. We imported 16 targets of the above three pathways into the String database, and the species was set to "Homo sapiens," thereby obtaining protein interactions. Subsequently, the PPI 
TABLE 1: The three centralities of 21 compounds were all higher than the average in C-T-D network.

\begin{tabular}{|c|c|c|c|c|c|}
\hline MOL ID & Molecule name & Structure & $\begin{array}{c}\text { Degree } \\
\text { centrality } \\
\text { (DC) }\end{array}$ & $\begin{array}{c}\text { Betweenness } \\
\text { centrality (BC) }\end{array}$ & Closeness centrality (CC) \\
\hline MOL000675 & Oleic acid & & 17 & 0.1394 & 0.4577 \\
\hline MOL002526 & $\delta$-Guaijene & & 8 & 0.0242 & 0.304 \\
\hline MOL000911 & Terpilene & & 8 & 0.0248 & 0.3916 \\
\hline MOL000126 & (-)-Nopinene & & 6 & 0.0398 & 0.4362 \\
\hline MOL000069 & Palmitic acid & & 6 & 0.0332 & 0.3779 \\
\hline MOL000118 & (L)-Alpha-terpineol & & 5 & 0.0119 & 0.3892 \\
\hline MOL000254 & Eugenol & & 5 & 0.0297 & 0.4305 \\
\hline MOL003521 & Isohomogenol & & 5 & 0.0083 & 0.3869 \\
\hline MOL000677 & $\begin{array}{c}\text { (1R, } 4 \mathrm{R}) \text {-4-Isopropyl-1,6- } \\
\text { dimethyltetralin }\end{array}$ & & 5 & 0.0067 & 0.3916 \\
\hline MOL002392 & Deltoin & & 5 & 0.0093 & 0.3916 \\
\hline MOL002417 & Fuzitine & & 5 & 0.0086 & 0.3801 \\
\hline
\end{tabular}


TABle 1: Continued.

\begin{tabular}{|c|c|c|c|c|c|}
\hline MOL ID & Molecule name & Structure & $\begin{array}{c}\text { Degree } \\
\text { centrality } \\
\text { (DC) }\end{array}$ & $\begin{array}{c}\text { Betweenness } \\
\text { centrality }(\mathrm{BC})\end{array}$ & Closeness centrality (CC) \\
\hline MOL000431 & Coumarin & & 5 & 0.0127 & 0.3757 \\
\hline MOL003525 & Pyruvophenone & & 5 & 0.0253 & 0.3631 \\
\hline MOL000198 & (R)-Linalool & & 4 & 0.0075 & 0.3736 \\
\hline MOL000131 & Linoleic acid & & 4 & 0.0181 & 0.414 \\
\hline MOL001393 & Myristic acid & & 4 & 0.0146 & 0.3693 \\
\hline MOL000932 & Alpha-farnesene & & 4 & 0.0121 & 0.3652 \\
\hline MOL000991 & Cinnamaldehyde & & 4 & 0.0121 & 0.3652 \\
\hline MOL002295 & Cinnamic acid & & 3 & 0.0141 & 0.4037 \\
\hline MOL000249 & Methylcinnamate & & 3 & 0.0141 & 0.4037 \\
\hline MOL003531 & 3-Methoxycinnamaldehyde & & 3 & 0.0141 & 0.4037 \\
\hline
\end{tabular}

network was further visualized and topology analyzed using Cytoscape 3.7.1 (see Figure 8). The network was constructed including 18 nodes and 67 edges. All the information of targets in PPI network was supplemented (see Table S2). The 8 target genes with the degree greater than their average score of connectivity were selected as the hub genes for 


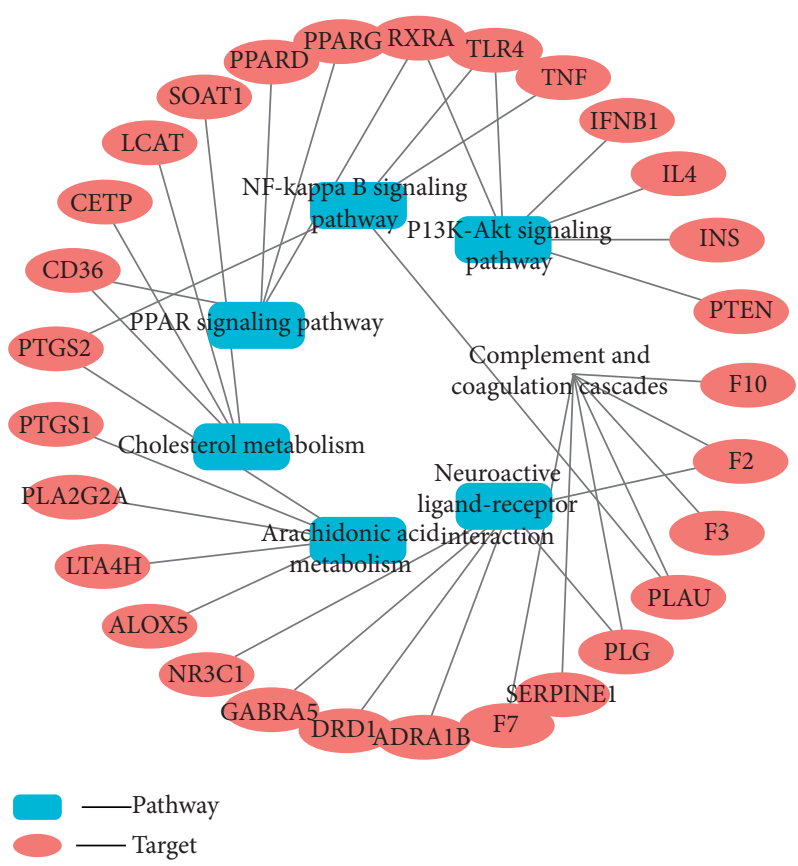

Figure 7: Target-pathways (T-P) network in treatment with CCVDs for rougui-fuzi. The pink ellipse represented the targets, and the blue rectangle represented the significant pathways of rougui-fuzi in treatment with CCVDs.

TABle 2: The 7 pathways information of rougui-fuzi in treatment with CCVDs.

\begin{tabular}{lccc}
\hline Pathways & Degree centrality (DC) & Betweenness centrality (BC) & Closeness centrality (CC) \\
\hline Complement and coagulation cascades & 7 & 0.5017 & 0.2381 \\
Neuroactive ligand-receptor interaction & 6 & 0.2193 & 0.1795 \\
PI3K-Akt signaling pathway & 6 & 0.5513 & 0.2518 \\
Arachidonic acid metabolism & 5 & 0.2185 & 0.2000 \\
Cholesterol metabolism & 4 & 0.1664 & 0.1515 \\
PPAR signaling pathway & 4 & 0.3479 & 0.1977 \\
NF-kappa B signaling pathway & 4 & 0.6673 & 0.2800 \\
\hline
\end{tabular}

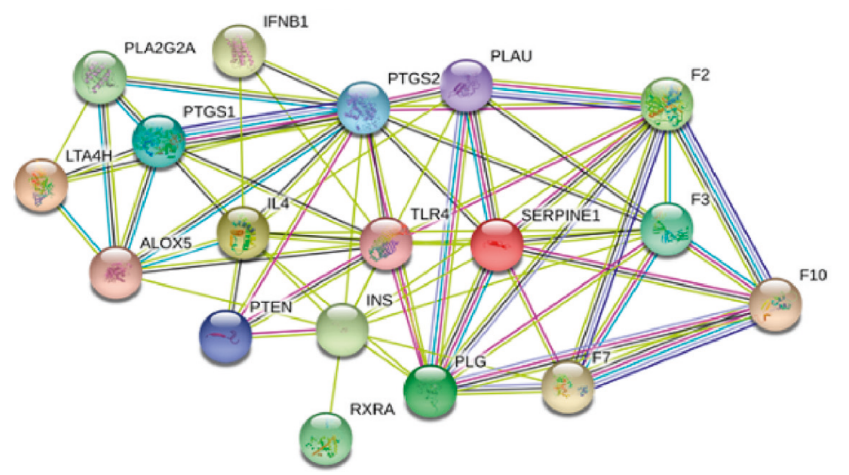

(a)

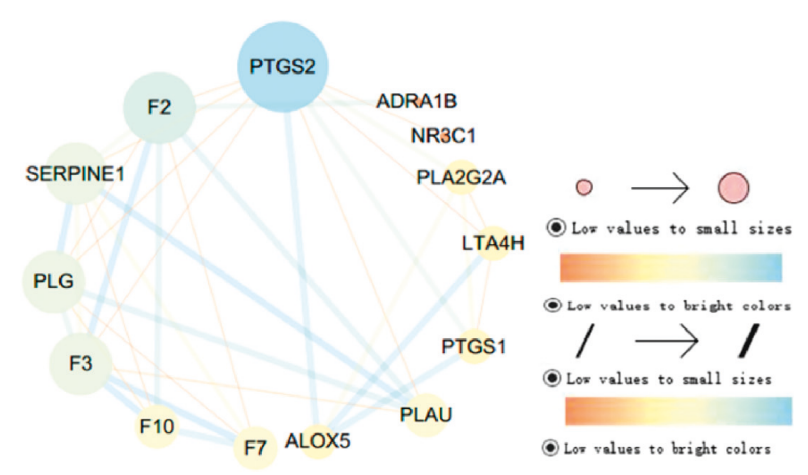

(b)

FIGURE 8: Protein-protein interaction (PPI) network by Cytoscape 3.7.1 software. The node size and color represent the size of the degree. Node size is proportional to its degree; node color is from orange to blue, and the corresponding degree gradually larger. Line thickness indicates the strength of data support. (a) PPI by string tool. (b) PPI by Cytoscape tool.

CCVDs (see Table 3), including PTGS2, IL-4, TLR4, F3, PLG, SERPINE1, and F2. According to literature research, PTGS2 rs20417CC genotype was significantly higher in patients with plaque compared with patients without plaque, and PTGS1 catalyses the conversion of arachidonic acid (AA) into prostaglandin $\mathrm{H} 2$ intermediates and thromboxane 
Table 3: 8 key target proteins obtained by PPI network.

\begin{tabular}{lccc}
\hline Gene name & Degree centrality (DC) & Betweenness centrality (BC) & Closeness centrality (CC) \\
\hline PTGS2 & 14 & 0.2411 & 0.8500 \\
INS & 12 & 0.1764 & 0.7727 \\
IL4 & 11 & 0.0701 & 0.7391 \\
TLR4 & 11 & 0.0734 & 0.7391 \\
F3 & 10 & 0.0361 & 0.7083 \\
PLG & 10 & 0.0361 & 0.7083 \\
SERPINE1 & 10 & 0.0361 & 0.7083 \\
F2 & 9 & 0.0275 & 0.6800 \\
\hline
\end{tabular}

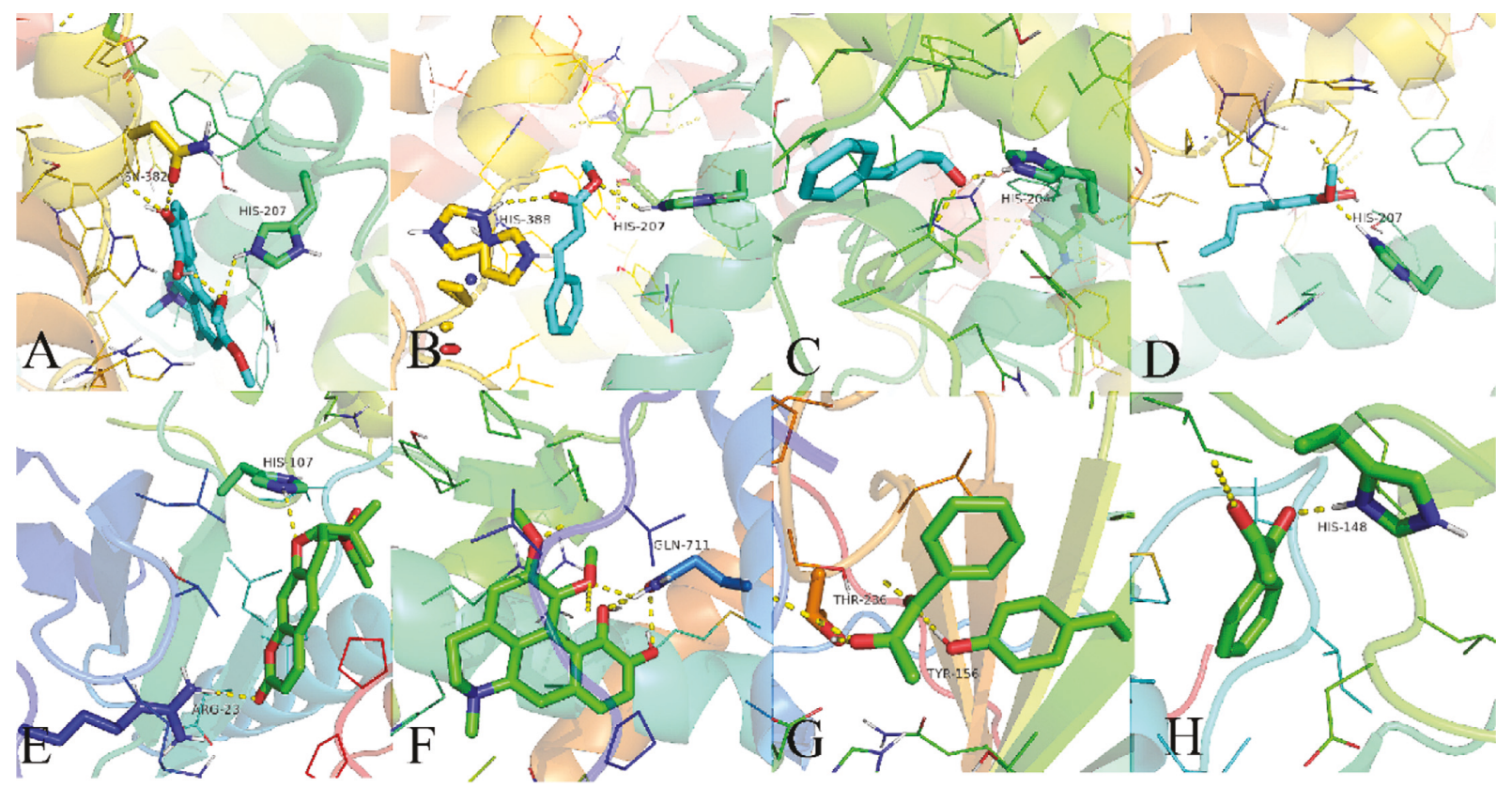

FIgUre 9: The conformations of some compounds and kernel targets: (a) PTGS2 with fuzitine, (b) PTGS2 with methylcinnamate, (c) PTGS2 with eugenol, (d) PTGS2 with cinnamaldehyde, (e) F2 with deltion, (f) AR with fuzitine, (g) F3 with pyruvophenone, and (h) F2 with pyruvophenone.

A2 (TxA2) [65]. Compared with healthy controls, hypertensive patients possess enhanced F2 generation, and diastolic blood pressure level of hypertensive patients is independently correlated with increased F2 generation [66]. PLG is a precursor of plasmin that degrades fibrin [67]. Plasminogen-dependent proteolysis has a beneficial effect during neurological recovery after stroke by facilitating axonal remodeling in the denervated spinal cord [68]. Based on the above, rougui-fuzi can regulate PTGS2, F2, and PLG in treatment with CCVDs.

3.9. Drug-Target Interaction Validation. The binding energy would have many results owing to different conformations during the molecular docking. In our previous studies, the potential protein targets of TCM were successfully predicted with a good compound-protein binding affinity based on the threshold level of $\leq-5.0 \mathrm{kcal} / \mathrm{mol}$. Thus, in the present work, target docking scores $\leq-5.0 \mathrm{kcal} / \mathrm{mol}$ as an empirical threshold were also selected as the potential targets for further analysis $[43,69]$. As a result, the partial compoundtarget interactions with binding energy $\leq-5.0 \mathrm{kcal} / \mathrm{mol}$ were shown vividly in (Figure 9), including (a) PTGS2 with fuzitine, (b) PTGS2 with methylcinnamate, (c) PTGS2 with eugenol, (d) PTGS2 with cinnamaldehyde, (e) F2 with deltion, (f) AR with fuzitine, (g) F3 with pyruvophenone, (h) F2 with pyruvophenone. A shows that fuzitine had hydrogen bonding contact with HIS-207 and ASN-382 residues of PTGS2. B shows that methylcinnamate had hydrogen bonding contact with HIS-388 and HIS-207 residues of PTGS2. Similarly, deltion also showed its interactions with ARG-23, HIS-107 residues of the target F2 in E.

3.10. UPLC-Q-Exactive-MSE of Rougui-Fuzi Extracts. For explore the active chemical of the rougui-fuzi, the high resolution spectrometry and the data of mass provided sufficient information. The data obtained from an online UPLC-QExactive and $\mathrm{MS}^{\mathrm{E}}$ satisfied the basic needs for the analysis of complex samples by recording exact mass data for each detectable compound and its structure. The herb components were detected and finally been confirmed by $\mathrm{MS}^{\mathrm{E}}$ substructure data of rougui-fuzi extracts as shown in Figure 10, including aconine, mesaconitine, benzoylhypaconine, 


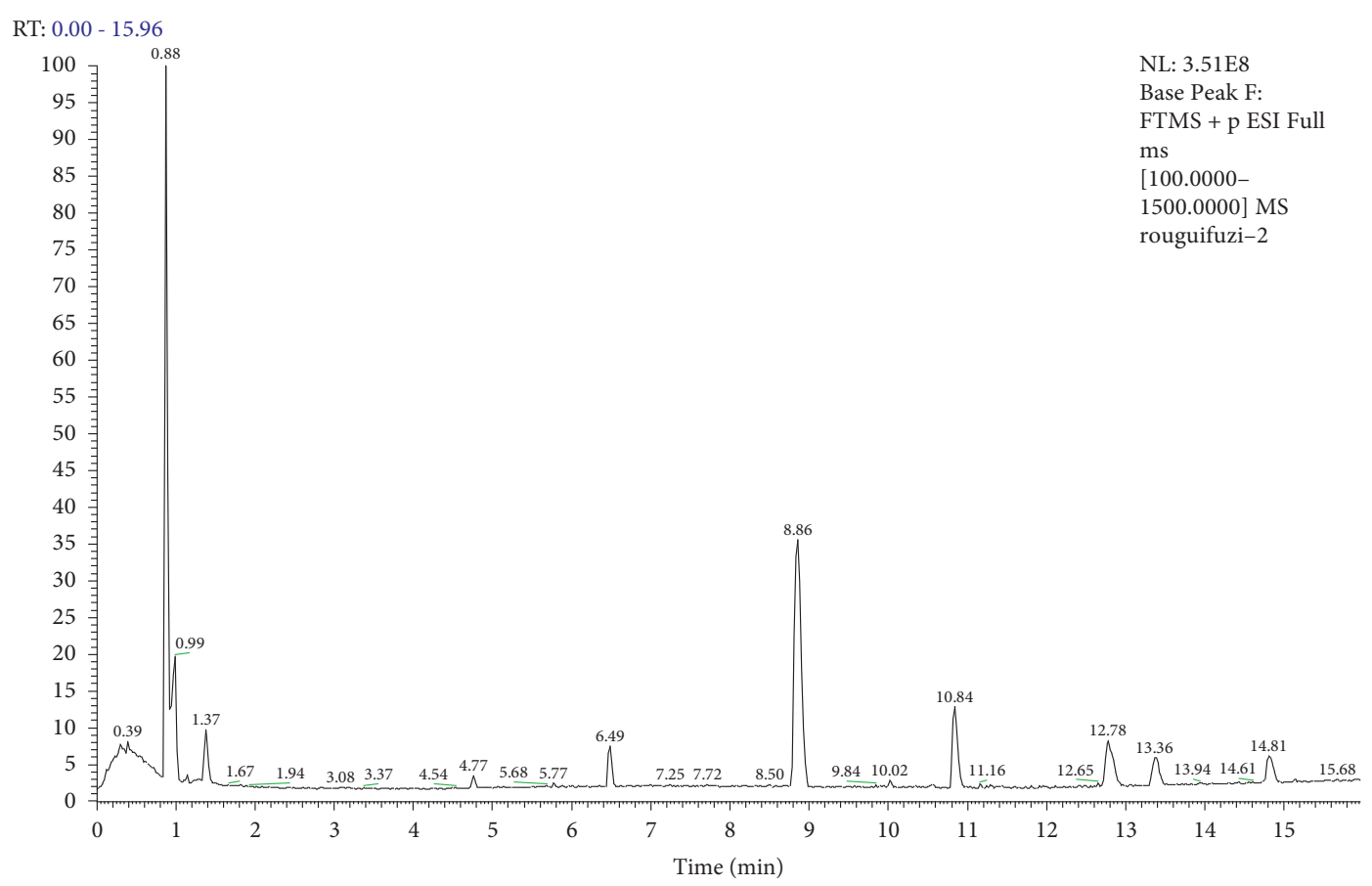

FIgURE 10: UPLC-Q-Exactive-MS ${ }^{\mathrm{E}}$ total ion current chromatogram of the extract of rougui-fuzi under positive ion mode.

TABLE 4: UPLC-Q-Exactive-MS ${ }^{\mathrm{E}}$ results of the bioactive ingredients in the extract of rougui-fuzi.

\begin{tabular}{|c|c|c|c|c|c|c|c|c|c|}
\hline Number & Compound & & Area & $\begin{array}{c}\mathrm{RT} \\
\text { (meas) }\end{array}$ & Formula & $\begin{array}{c}\text { Measured } \\
\mathrm{m} / \mathrm{z}\end{array}$ & Fragment ion & $\begin{array}{c}\text { Delta } \\
\mathrm{m} / \mathrm{z}\end{array}$ & Adducts \\
\hline 1 & Senbusine C & + & $1.2 E+08$ & 6.49 & C24H39NO7 & 454.28 & $\begin{array}{c}436.2688,404.2440 \\
409.6704\end{array}$ & -0.05 & $\mathrm{H}+$ \\
\hline 2 & Aconine & + & 7550000 & 5.73 & C25H41NO9 & 500.285 & $\begin{array}{c}154.9902,182.9852 \\
226.9514\end{array}$ & 0.53 & $\mathrm{H}+$ \\
\hline 3 & Cinnamic acid & + & 2260000 & 10.46 & $\mathrm{C} 9 \mathrm{H} 8 \mathrm{O} 2$ & 149.06 & $\begin{array}{c}167.1029,362.9263 \\
243.9417\end{array}$ & 0.6 & $\mathrm{H}+$ \\
\hline 4 & Coumarin & + & $8.2 E+07$ & 10.55 & $\mathrm{C} 9 \mathrm{H} 6 \mathrm{O} 2$ & 147.044 & $\begin{array}{c}154.9902,167.0128 \\
226.9513\end{array}$ & 0.36 & $\mathrm{H}+$ \\
\hline 5 & Mesaconitine & + & 79300 & 12.07 & C33H45NO11 & 632.307 & $\begin{array}{c}124.0870,154.9902 \\
167.0128\end{array}$ & 2.86 & $\mathrm{H}+$ \\
\hline 6 & Benzoylhypaconine & + & $3.3 E+07$ & 12.23 & C31H43NO9 & 574.301 & $\begin{array}{c}226.9514,154.9903 \\
362.9263\end{array}$ & 0.15 & $\mathrm{H}+$ \\
\hline 7 & Hypaconitine & + & 636000 & 12.49 & $\mathrm{C} 33 \mathrm{H} 45 \mathrm{NO} 10$ & 616.312 & $\begin{array}{c}226.9513,124.0869 \\
167.0128\end{array}$ & -0.26 & $\mathrm{H}+$ \\
\hline 8 & Cinnamaldehyde & + & $2 E+08$ & 13.36 & $\mathrm{C} 9 \mathrm{H} 8 \mathrm{O}$ & 133.065 & $\begin{array}{c}133.0648,154.9903 \\
167.0129\end{array}$ & 0.45 & $\mathrm{H}+$ \\
\hline
\end{tabular}

hypaconitine, cinnamic acid, cinnamaldehyde and coumarin, and senbusine $c$. Among them, cinnamic acid, cinnamaldehyde, and coumarin are contained in the 21 key compounds that are obtained based on the C-T-D network for rougui-fuzi in treatment with CCVDs. The retention times, molecular formulas, molecular ion peaks, adduct ions, and cleavage fragments of the identified compounds are provided in Table 4 .

3.11. GC-MS/MS Analysis of Rougui-Fuzi Extracts. The total ion chromatography (TIC) of essential oil of rougui-fuzi is shown in Figure 11. The analysis of GC-MS/MS data was performed using GC-MS solution software packages included with NIST14 database. 12 volatile compounds were identified based on the NIST 14 mass library by similarity searches (see Table 5), such as pyruvophenone, (L)-alphaterpineol, cinnamaldehyde, copaene, alpha-humulene, guaiene, alpha-cubebene, 3-methoxycinnamaldehyde, $\delta$-guaijene, T-cadinol, T-muurolol, and oleic acid. All these compounds belong to the 84 potential active ingredients that are obtained by TCMSP, and oleic acid, $\delta$-guaijene, terpilene, (L)-alpha-terpineol, pyruvophenone, cinnamaldehyde, and 3-methoxycinnamaldehyde are contained in the 21 key compounds that are obtained based on the C-T-D network 


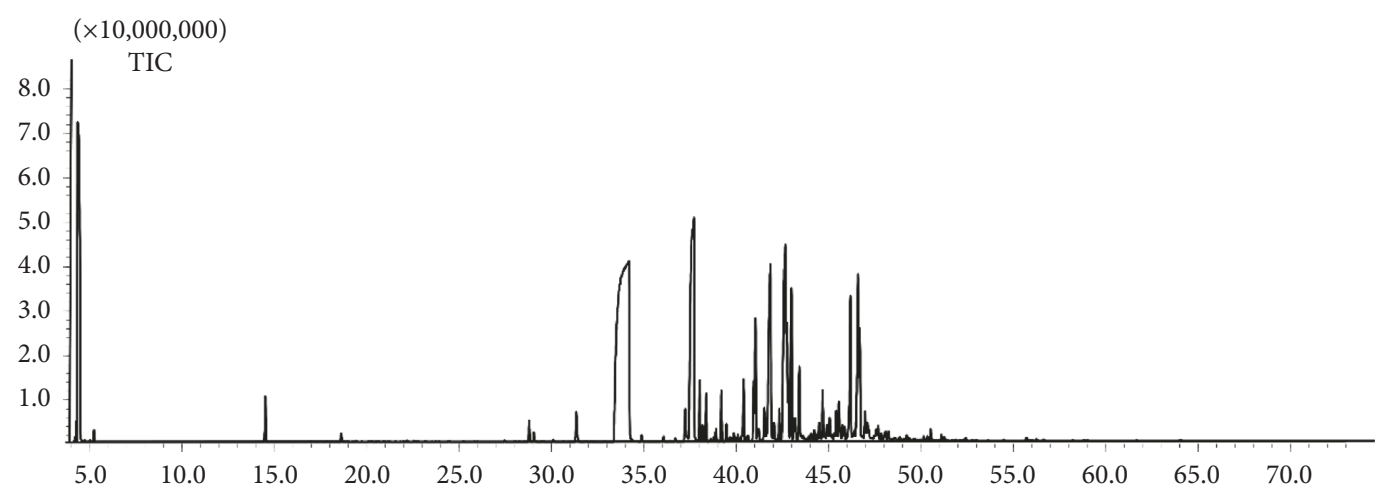

FIgURE 11: GC-MS total ion current chromatogram of the extract of rougui-fuzi.

TABLE 5: GC-MS results of the bioactive components in the extract of rougui-fuzi.

\begin{tabular}{|c|c|c|c|c|c|}
\hline Number & RT (meas) & Compound & Formula & Molecular weight & Matching accuracy \\
\hline 1 & 18.502 & Pyruvophenone & C9H80 & 132.15 & 87 \\
\hline 2 & 31.402 & (L)-Alpha-terpineol & $\mathrm{C} 10 \mathrm{H} 180$ & 154.24 & 87 \\
\hline 3 & 33.69 & Cinnamaldehyde & С9H8O & 132.15 & 96 \\
\hline 4 & 37.633 & Copaene & $\mathrm{C} 15 \mathrm{H} 24$ & 204.35 & 95 \\
\hline 5 & 40.342 & Alpha-humulene & $\mathrm{C} 15 \mathrm{H} 24$ & 204.35 & 92 \\
\hline 6 & 41.463 & Guaiene & $\mathrm{C} 15 \mathrm{H} 24$ & 204.35 & 90 \\
\hline 7 & 42.414 & Alpha-cubebene & $\mathrm{C} 15 \mathrm{H} 24$ & 204.35 & 90 \\
\hline 8 & 42.816 & 3-Methoxycinnamaldehyde & $\mathrm{C} 10 \mathrm{H} 10 \mathrm{O} 2$ & 162.19 & 85 \\
\hline 9 & 43.311 & $\delta$-Guaijene & $\mathrm{C} 15 \mathrm{H} 24$ & 204.35 & 88 \\
\hline 10 & 46.447 & T-Cadinol & $\mathrm{C} 15 \mathrm{H} 24$ & 204.35 & 94 \\
\hline 11 & 46.518 & T-Muurolol & $\mathrm{C} 15 \mathrm{H} 24$ & 204.35 & 90 \\
\hline 12 & 56.383 & Oleic acid & $\mathrm{C} 18 \mathrm{H} 34 \mathrm{O} 2$ & 282.46 & 80 \\
\hline
\end{tabular}

for rougui-fuzi in treatment with CCVDs. As a result, 10 of 21 key compounds have been verified by UPLC-Q-Exactive$\mathrm{MS}^{\mathrm{E}}$ and GC-MS/MS.

\section{Conclusions}

In summary, we used system pharmacology method to analyze the multicomponent, multitarget, multichannel mechanism for rougui-fuzi in treatment of CCVDs, including compound database construction, target fishing, disease mapping, network construction, and analysis. As a result, 21 key compounds, 8 key targets, and 3 key pathways were obtained for rougui-fuzi. Moreover, we performed molecular docking, which validated the reliability of the interactions of chemical compounds and targets based on systems pharmacology. Finally, we used UPLC-Q-Exactive$\mathrm{MS}^{\mathrm{E}}$ and GC-MS to analyze the potential ingredients of rougui-fuzi in the treatment of CCVDs. Collectively, herbal medicines always contain huge numbers of ingredients, diversified bioactive compounds of TCM with their related multiple targets, and multiple pathways, making it difficult to uncover the mechanisms of their interactions employing only traditional experimental approaches [70]. Systems pharmacology can offer an integrated system-level method to identify multichemicals, multitargets, and multipathways of Chinese herbs, which provided an alternative to understanding the scientific therapeutic mechanisms of Chinese herbs in treating complex diseases [21, 71].

\section{Data Availability}

The data used to support the findings of this study are included in the Supplementary Materials.

\section{Conflicts of Interest}

The authors declare that there are no conflicts of interest.

\section{Acknowledgments}

This work was supported by the study of Innovation Capability Support Program of Shaanxi (Program No. 2017KCT-27), Shannxi Research Centre on Discovery and Innovation of New Medicine (Program No. 2017YWZX-02), Key Research and Development Program of Shaanxi (Program No. 2018ZDXM-SF-003), and Department of Science and Technology of Shaanxi Province Projects (Program No. 2017SF-297).

\section{Supplementary Materials}

Table S1: 84 active ingredients of rougui-fuzi in CCVDrelated diseases. Table S2: 42 potential targets of rougui-fuzi in CCVD-related diseases. Table S3: the three centralities of potential targets in the PPI network. (Supplementary Materials) 


\section{References}

[1] K. Chang, Z. Jiang, C. Liu, J. Ren, T. Wang, and J. Xiong, "The Effects of CYP2C19 genotype on the susceptibility for nephrosis in cardio-cerebral vascular disease treated by anticoagulation,” Medicine, vol. 95, Article ID e4954, 2016.

[2] T. Zhang, K. Yu, and X. Li, "Cytochrome P450 family 4 subfamily F member 2 (CYP4F2) rs1558139, rs2108622 polymorphisms and susceptibility to several cardiovascular and cerebrovascular diseases," BMC Cardiovascular Disorders, vol. 18, p. 1, 2018.

[3] W. Williamson, A. J. Lewandowski, L. Griffanti et al., "Association of cardiovascular risk factors with MRI indices of cerebrovascular structure and function and white matter hyperintensities in young adults," JAMA, vol. 320, no. 7, pp. 665-673, 2018.

[4] Y. Gong, X. Cheng, C. Li et al., "Metabolic diseases and sex hormone levels affect differently in the development of cardiovascular and cerebrovascular diseases in elderly males," The Aging Male, vol. 18, no. 1, pp. 27-33, 2015.

[5] X. Feng, Y. Li, Y. Wang et al., "Danhong Injection in cardiovascular and cerebrovascular diseases: cardiovascular actions, molecular mechanisms, and therapeutic potential," Pharmacological Research, vol. 139, pp. 62-75, 2018.

[6] A. Squizzato, M. Bellesini, A. Takeda, S. Middeldorp, and M. P. Donadini, "Clopidogrel plus aspirin versus aspirin alone for preventing cardiovascular events," The Cochrane Database of Systematic Reviews, vol. 12, no. 12, Article ID CD005158, 2017.

[7] H. Blessberger, J. Kammler, H. Domanovits et al., "Perioperative beta-blockers for preventing surgery-related mortality and morbidity," The Cochrane Database of Systematic Reviews, vol. 3, no. 3, Article ID CD004476, 2018.

[8] A. Cynthia and T. Dennis, "MSc medication Co-payment vouchers, adherence with antiplatelet therapy, and adverse cardiovascular events after myocardial infarction," JAMA, vol. 321, pp. 37-39, 2019.

[9] M. Hong, S. Li, H. Tan et al., "A Network-Based Pharmacology Study of the Herb-Induced Liver Injury Potential of Traditional Hepatoprotective Chinese Herbal Medicines," Molecules, vol. 22, p. 632, 2017.

[10] X. Wang, X. Xu, W. Tao, Y. Li, Y. Wang, and L. Yang, “A systems biology approach to uncovering pharmacological synergy in herbal medicines with applications to cardiovascular disease," Evidence-Based Complementary and Alternative Medicine, vol. 2012, Article ID 519031, 15 pages, 2012.

[11] T. Pei, C. Zheng, C. Huang et al., "Systematic understanding the mechanisms of vitiligo pathogenesis and its treatment by Qubaibabuqi formula," Journal of Ethnopharmacology, vol. 190, pp. 272-287, 2016.

[12] H. Pan, "The theory of anti blood stasis of Cinnamomum cassia Presl and Aconitum carmichaelii Debx," Journal of Shanghai University of Traditional Chinese Medicine, vol. 4, pp. 34-37, 2006.

[13] H. Mou, P. Tu, and Y. Jiang, "Network pharmacology-based study on mechanisms of warming channel and relieving stagnation effects of Cinnamomum cassia Presl," Acta Pharmaceutica Sinica, vol. 51, pp. 1724-1733, 2016.

[14] X. Chen, C. Liu, N. Ma, T. Zhang, and Y. Deng, "Research progress on chemical constituents, pharmacological action and comprehensive application of Cinnamomum cassia Presl," Chinese Pharmacy, vol. 2018, pp. 2581-2584, 2018.

[15] X. Hou, E. Hao, J. Qin, J. Wei, and J. Jiao, "The analysis of chemical constituents, pharmacological action and Q-marker of Cinnamomum cassia Presl," Chinese Herbal Medicine, vol. 49, pp. 20-34, 2018.

[16] B.-C. Liao, C.-W. Hsieh, Y.-C. Liu, T.-T. Tzeng, Y.-W. Sun, and B.-S. Wung, "Cinnamaldehyde inhibits the tumor necrosis factor- $\alpha$-induced expression of cell adhesion molecules in endothelial cells by suppressing NF- $\kappa \mathrm{B}$ activation: effects upon I $\kappa \mathrm{B}$ and Nrf2," Toxicology and Applied Pharmacology, vol. 229, no. 2, pp. 161-171, 2008.

[17] L. Huang, T. Huang, R. Lin, W. Zheng, and W. Li, "Research progress of polysaccharide from aconite seed on delaying myocardial senescence," Chinese Journal of Clinical Pharmacology, vol. 34, pp. 2894-2896, 2018.

[18] L. Xie, E. J. Draizen, and P. E. Bourne, "Harnessing big data for systems pharmacology," Annual Review of Pharmacology and Toxicology, vol. 57, no. 1, pp. 245-262, 2017.

[19] C. Huang, C. Zheng, Y. Li, Y. Wang, A. Lu, and L. Yang, "Systems pharmacology in drug discovery and therapeutic insight for herbal medicines," Briefings in Bioinformatics, vol. 15, no. 5, pp. 710-733, 2013.

[20] W. Zhang, Y. Huai, Z. Miao, A. Qian, and Y. Wang, "Systems pharmacology for investigation of the mechanisms of action of traditional Chinese medicine in drug discovery," Frontiers in Pharmacology, vol. 10, p. 734, 2019.

[21] C. Ma, C. Zhai, T. Xu et al., "A systems pharmacology-based study of the molecular mechanisms of san cao decoction for treating hypertension," Evidence-Based Complementary and Alternative Medicine, vol. 2019, pp. 1-10, 2019.

[22] J. Zhang, R. Liang, L. Wang, and B. Yang, "Effects and mechanisms of Danshen-Shanzha herb-pair for atherosclerosis treatment using network pharmacology and experimental pharmacology," Journal of Ethnopharmacology, vol. 229, pp. 104-114, 2018.

[23] T. Hart and L. Xie, "Providing data science support for systems pharmacology and its implications to drug discovery," Expert Opinion on Drug Discovery, vol. 11, no. 3, pp. 241-256, 2016.

[24] W. Chen, C. Huang, C. Yang et al., "Danggui sini decoction protected islet endothelial cell survival from hypoxic damage via PI3K/Akt/eNOS pathway," Evidence-Based Complementary and Alternative Medicine, vol. 2018, pp. 1-10, 2018.

[25] Q. Wu, C. Cai, P. Guo et al., "In silico identification and mechanism exploration of hepatotoxic ingredients in traditional Chinese medicine," Frontiers in Pharmacology, vol. 10, p. 458, 2019.

[26] J. Ru, P. Li, J. Wang et al., "TCMSP: a database of systems pharmacology for drug discovery from herbal medicines," Journal of Cheminformatics, vol. 6, p. 13, 2014.

[27] Y. Li, J. Zhang, L. Zhang et al., "Systems pharmacology to decipher the combinational anti-migraine effects of Tianshu formula," Journal of Ethnopharmacology, vol. 174, pp. 45-56, 2015.

[28] W. Tao, X. Xu, X. Wang et al., "Network pharmacology-based prediction of the active ingredients and potential targets of Chinese herbal Radix Curcumae formula for application to cardiovascular disease," Journal of Ethnopharmacology, vol. 145 , no. 1, pp. 1-10, 2013.

[29] J. S. Amberger, C. A. Bocchini, F. Schiettecatte, A. F. Scott, and A. Hamosh, "OMIM.org: online Mendelian Inheritance in Man (OMIM), an online catalog of human genes and genetic disorders," Nucleic Acids Research, vol. 43, no. D1, pp. D789-D798, 2014.

[30] J. M. Barbarino, M. Whirl-Carrillo, R. B. Altman, and T. E. Klein, "PharmGKB: a worldwide resource for pharmacogenomic information," Wiley Interdisciplinary Reviews: 
Systems Biology and Medicine, vol. 10, no. 4, Article ID e1417, 2018.

[31] W. Wang, T. Liu, L. Yang et al., "Study on the multi-targets mechanism of triphala on cardio-cerebral vascular diseases based on network pharmacology," Biomedicine \& Pharmacotherapy, vol. 116, Article ID 108994, 2019.

[32] N. Wang, F. Zhu, M. Shen et al., "Network pharmacologybased analysis on bioactive anti-diabetic compounds in Potentilla discolor bunge," Journal of Ethnopharmacology, vol. 241, Article ID 111905, 2019.

[33] A. Taskiran-Sag, M. Yemisci, Y. Gursoy-Ozdemir et al., "Improving microcirculatory reperfusion reduces parenchymal oxygen radical formation and provides neuroprotection," Stroke, vol. 49, pp. 1267-1275, 2018.

[34] M. Bruschettini, O. Romantsik, S. Zappettini, R. Banzi, L. Ramenghi, and M. G. Calevo, "Heparin for the prevention of intraventricular haemorrhage in preterm infants," Cochrane Database of Systematic Reviews, vol. 3, Article ID CD011636, 2016.

[35] Y.-P. Zhang, C.-S. Pan, L. Yan et al., "Catalpol restores LPSelicited rat microcirculation disorder by regulation of a network of signaling involving inhibition of TLR-4 and SRC," American Journal of Physiology-Gastrointestinal and Liver Physiology, vol. 311, no. 6, pp. G1091-G1104, 2016.

[36] S. Banerjee, P. Bhattacharjee, A. Kar, and P. K. Mukherjee, "LC-MS/MS analysis and network pharmacology of Trigonella foenum-graecum-a plant from Ayurveda against hyperlipidemia and hyperglycemia with combination synergy," Phytomedicine, vol. 60, Article ID 152944, 2019.

[37] Y. Liang, X. Zhang, J. Zou et al., "Pharmacology mechanism of Flos magnoliae and Centipeda minima for treating Allergic Rhinitis based on Pharmacology network," Drug Development and Industrial Pharmacy, vol. 45, no. 9, pp. 1547-1555, 2019.

[38] C. Zheng, T. Pei, C. Huang et al., "A novel systems pharmacology platform to dissect action mechanisms of traditional Chinese medicines for bovine viral diarrhea disease," European Journal of Pharmaceutical Sciences, vol. 94, pp. 33-45, 2016.

[39] N. T. Doncheva, J. H. Morris, J. Gorodkin, and L. J. Jensen, "Cytoscape stringApp: network analysis and visualization of proteomics data," Journal of Proteome Research, vol. 18, no. 2, pp. 623-632, 2018.

[40] A. Y. Lee, W. Park, T.-W. Kang, M. H. Cha, and J. M. Chun, "Network pharmacology-based prediction of active compounds and molecular targets in Yijin-Tang acting on hyperlipidaemia and atherosclerosis," Journal of Ethnopharmacology, vol. 221, pp. 151-159, 2018.

[41] M. Su, C. Guo, M. Liu, X. Liang, and B. Yang, "Therapeutic targets of vitamin $\mathrm{C}$ on liver injury and associated biological mechanisms: a study of network pharmacology," International Immunopharmacology, vol. 66, pp. 383-387, 2019.

[42] B. Li, J. Rui, X. Ding, and X. Yang, "Exploring the multicomponent synergy mechanism of Banxia Xiexin Decoction on irritable bowel syndrome by a systems pharmacology strategy," Journal of Ethnopharmacology, vol. 233, pp. 158168, 2019.

[43] W. Zhou, J. Wang, Z. Wu, C. Huang, A. Lu, and Y. Wang, "Systems pharmacology exploration of botanic drug pairs reveals the mechanism for treating different diseases," Scientific Reports, vol. 6, no. 1, 2016.

[44] M. Tang, L. Zhao, and M. Xu, "Research progress on chemical constituents and pharmacological effects of aconite," Guangxi Plants, vol. 37, pp. 1614-1627, 2017.
[45] S. Cheng, X. Zhang, Q. Feng et al., “Astragaloside IV exerts angiogenesis and cardioprotection after myocardial infarction via regulating PTEN/PI3K/Akt signaling pathway," Life Sciences, vol. 227, pp. 82-93, 2019.

[46] C. Li, Y. Zhang, Q. Wang et al., "Dragon's Blood exerts cardioprotection against myocardial injury through PI3K-AKTmTOR signaling pathway in acute myocardial infarction mice model," Journal of Ethnopharmacology, vol. 227, pp. 279-289, 2018.

[47] X. Li, X. Xu, and Y. Wang, "A system-level investigation into the mechanisms of Chinese traditional medicine: compound danshen formula for cardiovascular disease treatment," PLoS One, vol. 7, Article ID e43918, 2012.

[48] J. Song and L. C. KimY, "Neuroprotective effects of oleic acid in rodent models of cerebral ischaemia," Scientific Reports, vol. 9, p. 10732, 2019.

[49] B. T. Steffen, D. Duprez, M. Szklo, W. Guan, and M. Y. Tsai, "Circulating oleic acid levels are related to greater risks of cardiovascular events and all-cause mortality: the MultiEthnic Study of Atherosclerosis," Journal of Clinical Lipidology, vol. 12, no. 6, pp. 1404-1412, 2018.

[50] C. L. Satizabal, C. Samieri, K. L. Davis-Plourde et al., "APOE and the association of fatty acids with the risk of stroke, coronary heart disease, and mortality," Stroke, vol. 49, no. 12, pp. 2822-2829, 2018.

[51] N. Ahmad, R. Ahmad, M. Alam, and F. Ahmad, "Quantification and brain targeting of eugenol-loaded surface modified nanoparticles through intranasal route in the treatment of cerebral ischemia," Drug Research, vol. 68, no. 10, pp. 584595, 2018.

[52] S. Akoudad, S. K. L. Darweesh, M. J. G. Leening et al., "Use of coumarin anticoagulants and cerebral microbleeds in the general population," Stroke, vol. 45, no. 11, pp. 3436-3439, 2014.

[53] T. Knecht, J. Story, J. Liu, W. Davis, C. Borlongan, and I. dela Peña, "Adjunctive therapy approaches for ischemic stroke: innovations to expand time window of treatment," International Journal of Molecular Sciences, vol. 18, no. 12, p. 2756, 2017.

[54] M. S. Herrera-Meza, M. R. Mendoza-López, O. García-Barradas et al., "Dietary anhydrous milk fat naturally enriched with conjugated linoleic acid and vaccenic acid modify cardiovascular risk biomarkers in spontaneously hypertensive rats," International Journal of Food Sciences and Nutrition, vol. 64, no. 5, pp. 575-586, 2013.

[55] E. T. M. Berends, A. Kuipers, M. M. Ravesloot, R. T. Urbanus, and S. H. M. Rooijakkers, "Bacteria under stress by complement and coagulation," FEMS Microbiology Reviews, vol. 38, no. 6, pp. 1146-1171, 2014.

[56] E. M. Conway, "Complement-coagulation connections," Blood Coagulation \& Fibrinolysis, vol. 29, no. 3, pp. 243-251, 2018.

[57] J. H. Foley, "Examining coagulation-complement crosstalk: complement activation and thrombosis," Thrombosis Research, vol. 141, pp. S50-S54, 2016.

[58] H. Meng, Y. Liu, and L. Lai, "Diverse ways of perturbing the human arachidonic acid metabolic network to control inflammation," Accounts of Chemical Research, vol. 48, no. 8, pp. 2242-2250, 2015.

[59] T. Sonnweber, A. Pizzini, M. Nairz, G. Weiss, and I. Tancevski, "Arachidonic acid metabolites in cardiovascular and metabolic diseases," International Journal of Molecular Sciences, vol. 19, no. 11, p. 3285, 2018. 
[60] H.-K. Chung, Y. Cho, H. J. Do, K. Oh, W.-K. Seo, and M.-J. Shin, "Plasma phospholipid arachidonic acid and lignoceric acid are associated with the risk of cardioembolic stroke," Nutrition Research, vol. 35, no. 11, pp. 1001-1008, 2015.

[61] S. Luo, H. Li, Z. Mo et al., "Connectivity map identifies luteolin as a treatment option of ischemic stroke by inhibiting MMP9 and activation of the PI3K/Akt signaling pathway," Experimental \& Molecular Medicine, vol. 51, p. 37, 2019.

[62] Q. Zhou, G. Meng, F. Teng, Q. Sun, and Y. Zhang, "Effects of astragalus polysaccharide on apoptosis of myocardial microvascular endothelial cells in rats undergoing hypoxia/ reoxygenation by mediation of the PI3K/Akt/eNOS signaling pathway," Journal of Cellular Biochemistry, vol. 119, no. 1, pp. 806-816, 2017.

[63] P. Shabanzadeh, M. D’Onofrio, M. Magharious, B. Choi, P. Monnier, and P. D. Koeberle, "Modifying PTEN recruitment promotes neuron survival, regeneration, and functional recovery after CNS injury," Cell Death \& Disease, vol. 10, 2019.

[64] Z.-H. Yu, M. Cai, J. au et al., "PI3K/Akt pathway contributes to neuroprotective effect of Tongxinluo against focal cerebral ischemia and reperfusion injury in rats," Journal of Ethnopharmacology, vol. 181, pp. 8-19, 2016.

[65] Z.-H. Yu, M. Cai, J. Xiang, Z.-N. Zhang, and J.-S. Zhang, "Genetic variants of PTGS2, TXA2R and TXAS1 are associated with carotid plaque vulnerability, platelet activation and TXA2 levels in ischemic stroke patients," PLoS One, vol. 12, no. 7, Article ID e0180704, 2017.

[66] C. Blanton, Z. He, T. Gottschall-Pass, and I. Sweeney, "Probiotics blunt the anti-hypertensive effect of blueberry feeding in hypertensive rats without altering hippuric acid production," PLoS One, vol. 10, Article ID e0142036, 2015.

[67] Y. Tashima, F. Banno, and Y. Kita, "Plasminogen Tochigi mice exhibit phenotypes similar to wild-type mice under experimental thrombotic conditions," PLoS One, vol. 12, Article ID e0180981, 2017.

[68] Z. Liu, Y. Li, and C. Qian, "Plasminogen deficiency causes reduced corticospinal axonal plasticity and functional recovery after stroke in mice," PLoS One, vol. 9, Article ID e94505, 2014.

[69] H. Liu, J. Wang, W. Zhou, Y. Wang, and L. Yang, "Systems approaches and polypharmacology for drug discovery from herbal medicines: an example using licorice," Journal of Ethnopharmacology, vol. 146, pp. 773-793, 2015.

[70] J. Wang, Y. Li, Y. Yang et al., "Systems pharmacology dissection of multiscale mechanisms of action for herbal medicines in treating rheumatoid arthritis," Molecular Pharmaceutics, vol. 14, no. 9, pp. 3201-3217, 2017.

[71] W. Zhou, Y. Wang, A. Lu, and G. Zhang, "Systems pharmacology in small molecular drug discovery," International Journal of Molecular Sciences, vol. 17, no. 2, p. 246, 2016. 


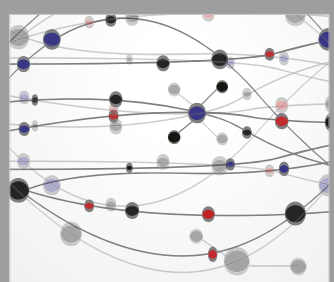

The Scientific World Journal
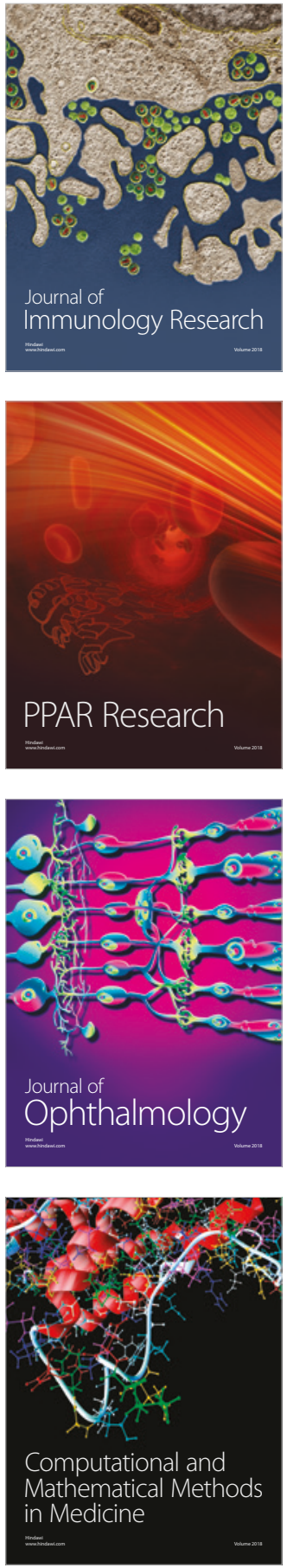

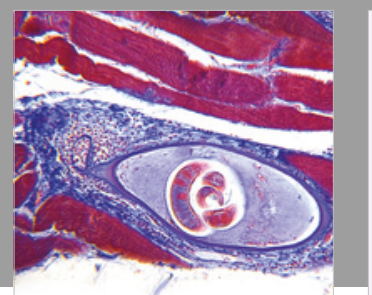

Gastroenterology Research and Practice

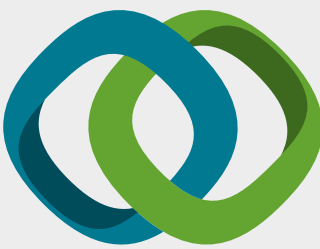

\section{Hindawi}

Submit your manuscripts at

www.hindawi.com
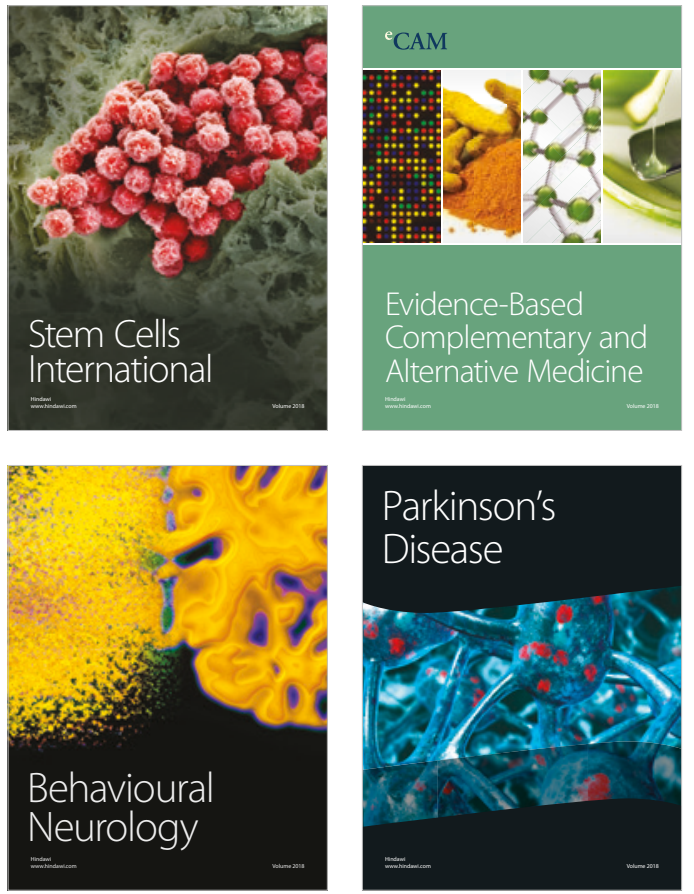

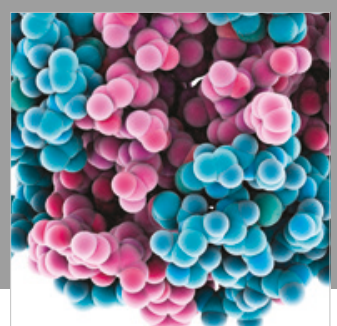

ournal of

Diabetes Research

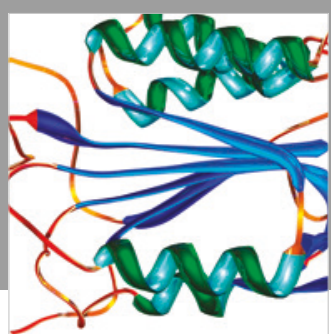

Disease Markers
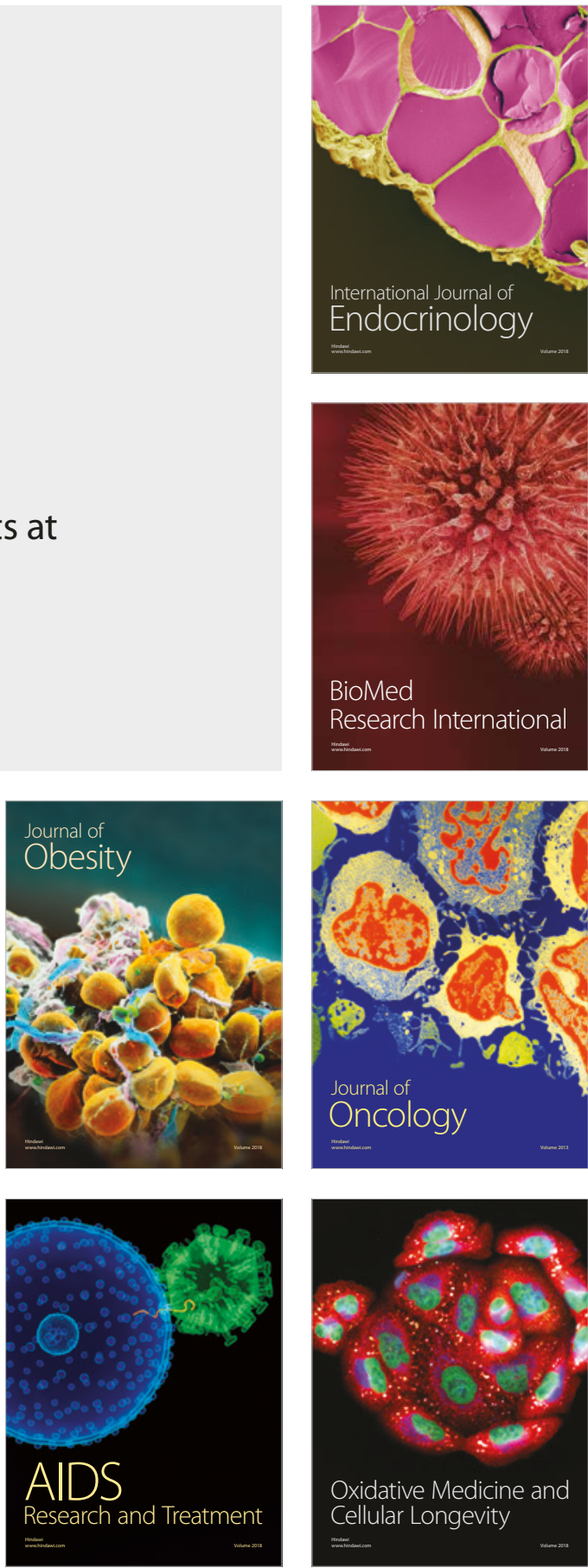